Supporting Information for

\title{
Effect of Nanoscale Confinement on Polymer Infiltrated Scaffold Metal Composites
}

Shawn M. Maguire ${ }^{1 \neq}$, Connor R. Bilchak ${ }^{1,2 \neq}$, John S. Corsi ${ }^{1}$, Samuel S. Welborn ${ }^{1}$, Theresa Tsaggaris ${ }^{1}$, Jamie Ford ${ }^{3}$, Eric Detsi ${ }^{1}$, Zahra Fakhraai ${ }^{2 *}$, Russell J. Composto ${ }^{1^{*}}$

1: Department of Materials Science and Engineering, University of Pennsylvania, Philadelphia, PA

2: Department of Chemistry, University of Pennsylvania, Philadelphia, PA

3: Nanoscale Characterization Facility, Singh Center for Nanotechnology, University of Pennsylvania, Philadelphia, PA

\#: These authors contributed equally.

*: co-corresponding authors to whom correspondence should be addressed: composto@seas.upenn.edu, fakhraai@sas.upenn.edu 


\section{Supporting Information Table of Contents}

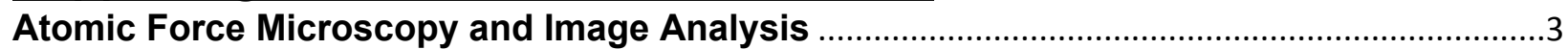

Theoretical Prediction of Polymer Chain Diffusion ............................................................

Ellipsometric Measurements and Modeling Procedure ........................................................10

In-situ Infiltration and Glass Transition Temperature Measurements ................................17

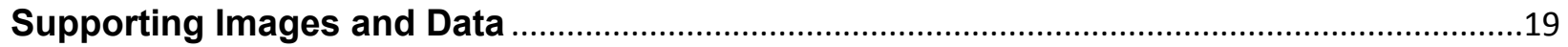




\section{Atomic Force Microscopy and Image Analysis}

Tapping mode AFM was performed using an Agilent 5420 AFM with non-contact tips (TAP300ALG-50 radius of curvature $<10 \mathrm{~nm}$, Ted Pella). Image filtering and analysis was performed using Gwyddion software. The images were leveled using the mean field subtraction function and scan rows aligned using a median filter. Background noise was removed using a $4^{\text {th }}$ order polynomial background. Average ligament dimensions were calculated by averaging the lengths and widths of at least 100 features each in five separate images. Radial autocorrelation functions were also performed to extract the characteristic dimension of the NP structure. Autocorrelation functions were performed on $2 \times 2 \mu \mathrm{m}^{2}$ images with filters and background subtraction as described above.

Figures S1-S7 show AFM images of the NPG-composites at various stages of processing used in this work. For unfilled NPG, the bicontinuous structure of gold ligaments and nanopores is clearly visible, with the pore diameter and gold ligament width increasing during thermal treatment at $175^{\circ} \mathrm{C}$. The unfilled materials also display a high surface roughness ( $\approx 25-30 \mathrm{~nm}$ ). Polymerinfiltrated NPG composites where the infiltration process has completed (i.e., the polymer chains have reached the surface of the NPG) conversely have low surface roughness, and the bicontinuous gold/pore structure becomes more difficult to discern by eye. Figures S6 and S7 show a series of AFM topography and phase images of NPG composites infiltrated with PS and P2VP chains, respectively. The infiltration procedure for each material involved thermal annealing at $150^{\circ} \mathrm{C}$ for 3 hours, followed by cooling to room temperature. The surface roughness remains relatively low $\left(<5 \mathrm{~nm}\right.$ ) for composites with chain $\mathrm{M}_{\mathrm{w}}<500 \mathrm{kDa}$, but increases substantially above this cut-off for both polymers. A more clearly visible ligament/pore structure also develops in tandem with an increase in surface roughness. We interpret these data as indicative of whether the polymer chains have obtained 'full infiltration' when subjected to a given thermal annealing procedure; in this case, composites with chain $\mathrm{M}_{\mathrm{w}}<500 \mathrm{kDa}$ fully infiltrate into the nanopores after 3 hours of annealing at $150^{\circ} \mathrm{C}$, while chains with larger $\mathrm{M}_{w}$ do not. We note that this analysis only probes the surface of the NPG film and hence cannot provide information on the remainder of the NPG film (e.g., if the polymer has infiltrated through the bottom $50 \mathrm{~nm}$ of the NPG).

A.

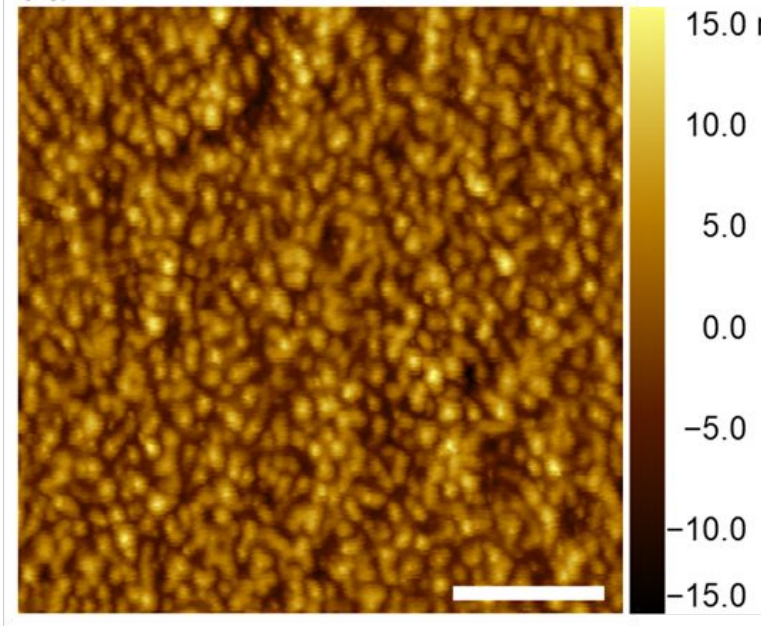

B.

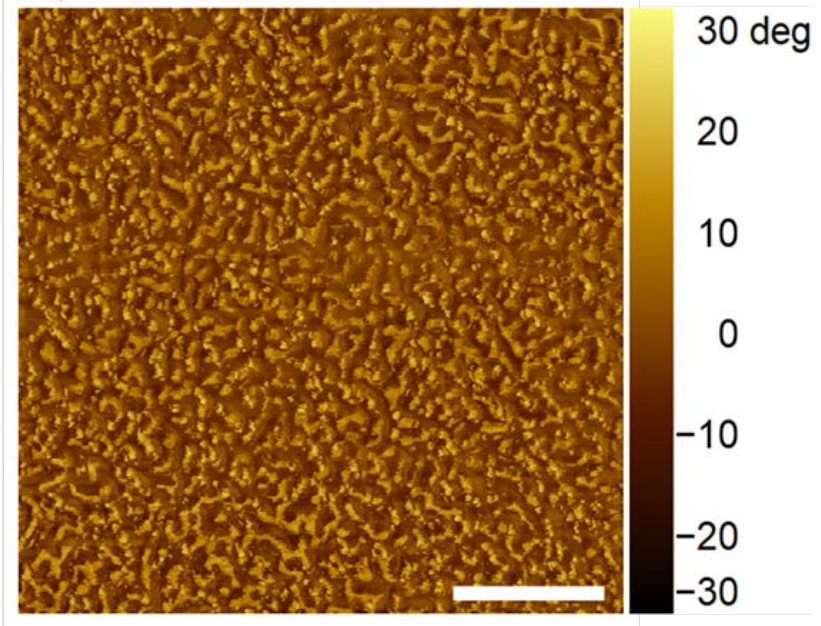

Figure S1: (A) amplitude and (B) phase image of the as-cast NPG. The scale bars are $500 \mathrm{~nm}$. 
A.

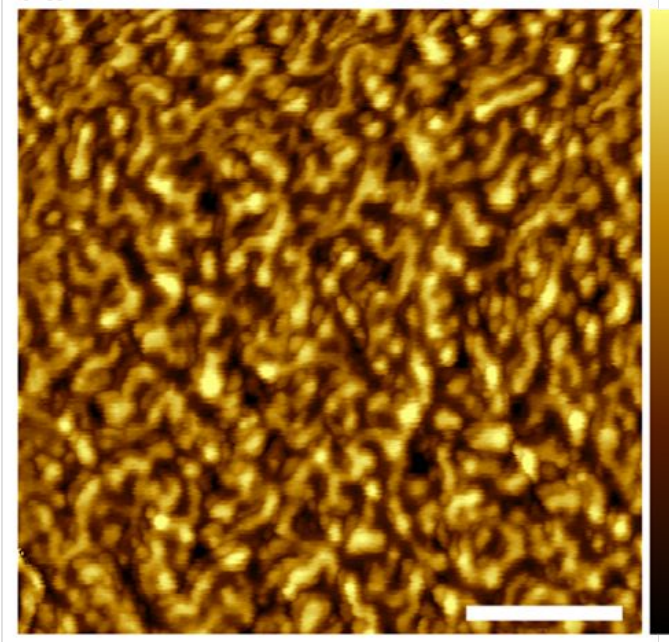

B.

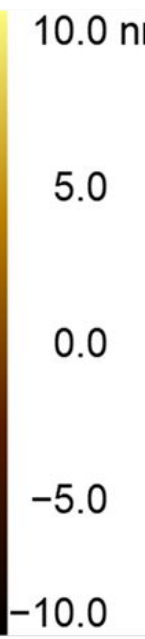

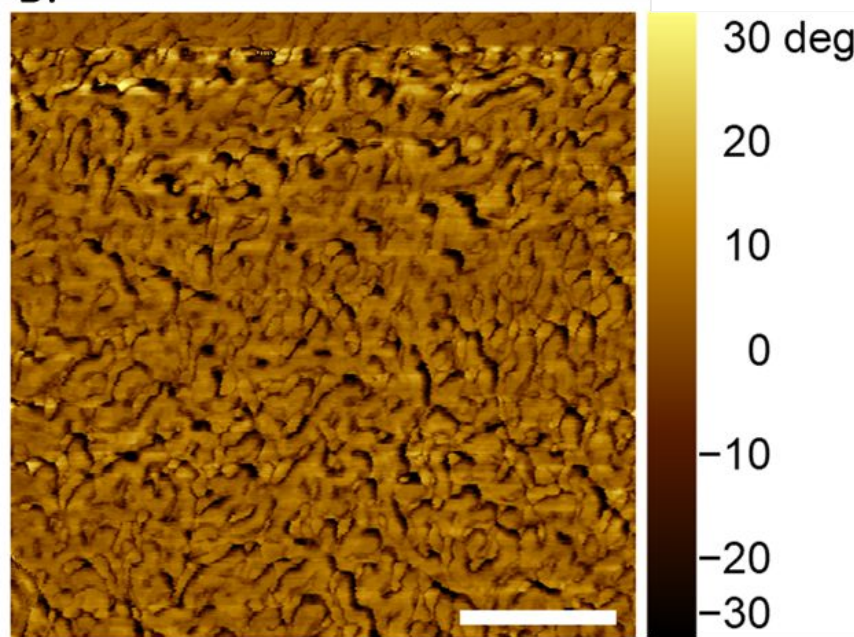

Figure S2: (A) amplitude and (B) phase image of the NPG after coarsening at $175^{\circ} \mathrm{C}$ for 3 hours. The scale bars are $500 \mathrm{~nm}$.

A.

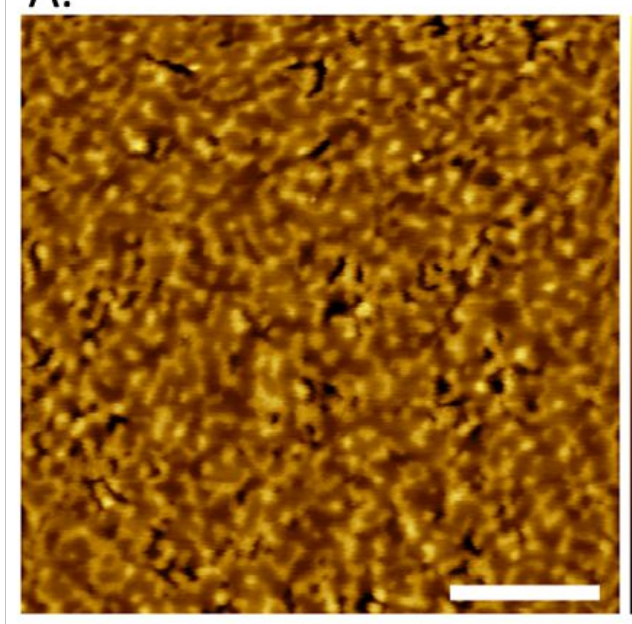

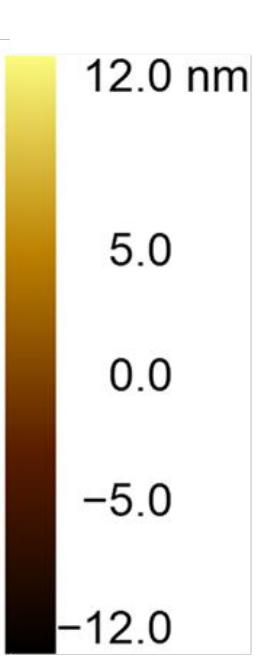

B.

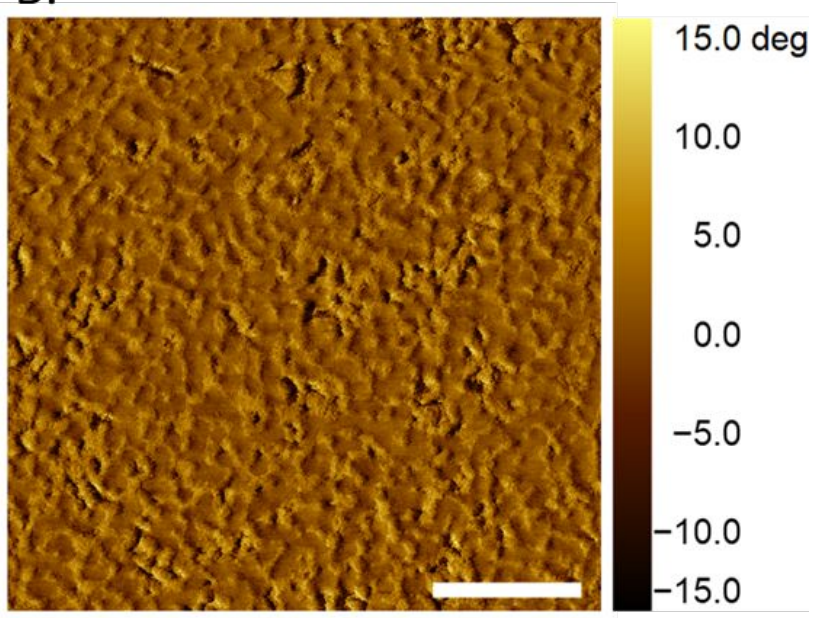

Figure S3: (A) amplitude and (B) phase image of the coarsened NPG after infiltrating with $62 \mathrm{kDa}$ PS. The scale bars are $500 \mathrm{~nm}$. 
A.

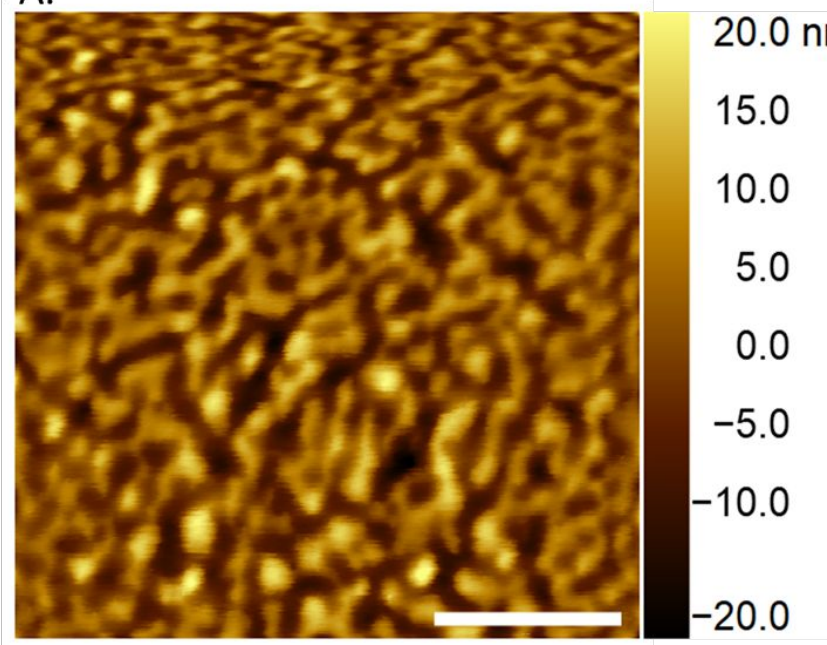

B.

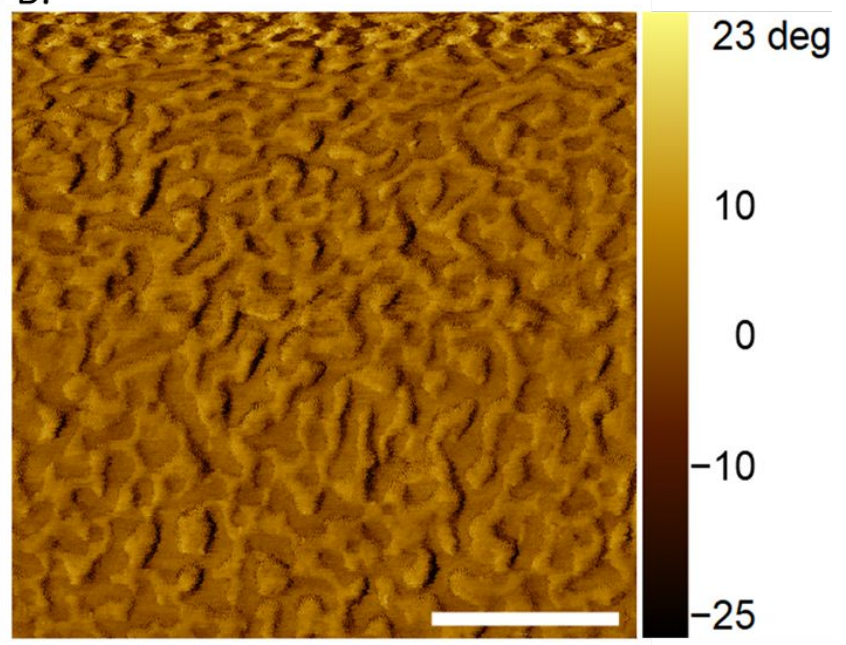

Figure S4: (A) amplitude and (B) phase image of the coarsened NPG after aging at ambient conditions for 6 months. The scale bars are $500 \mathrm{~nm}$.

A.

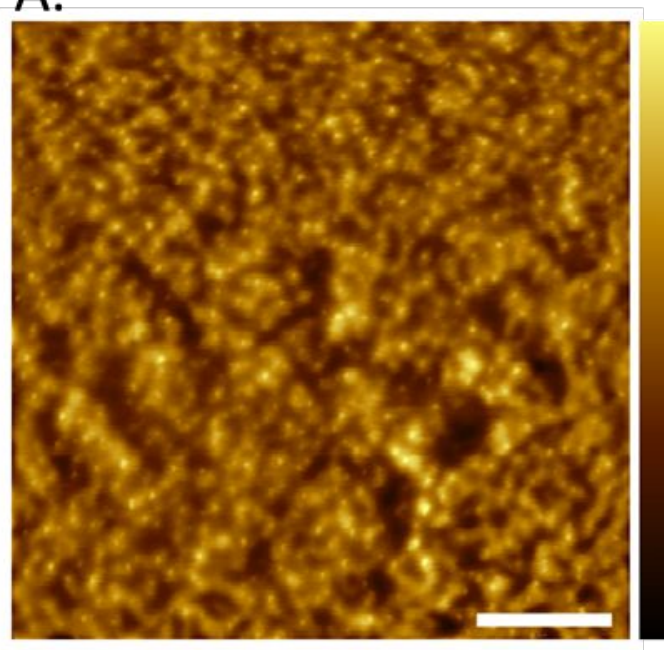

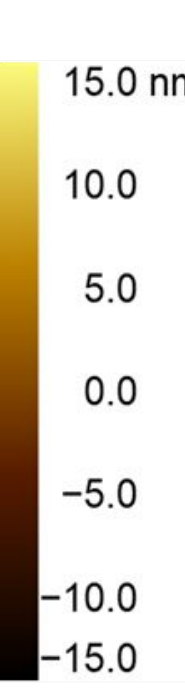

B.

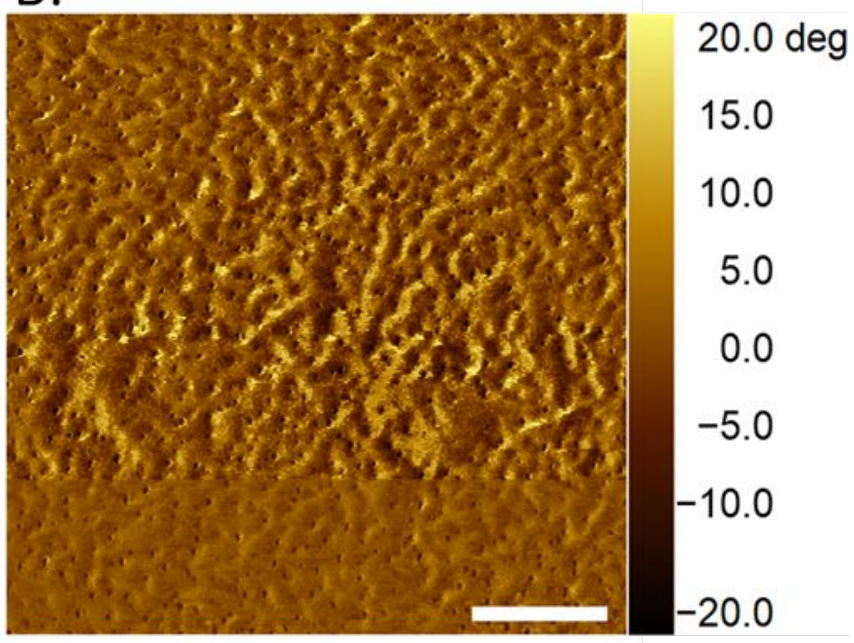

Figure S5: (A) amplitude and (B) phase image of the infiltrated NPG composite after aging at ambient conditions for 6 months. The scale bars are $500 \mathrm{~nm}$. 


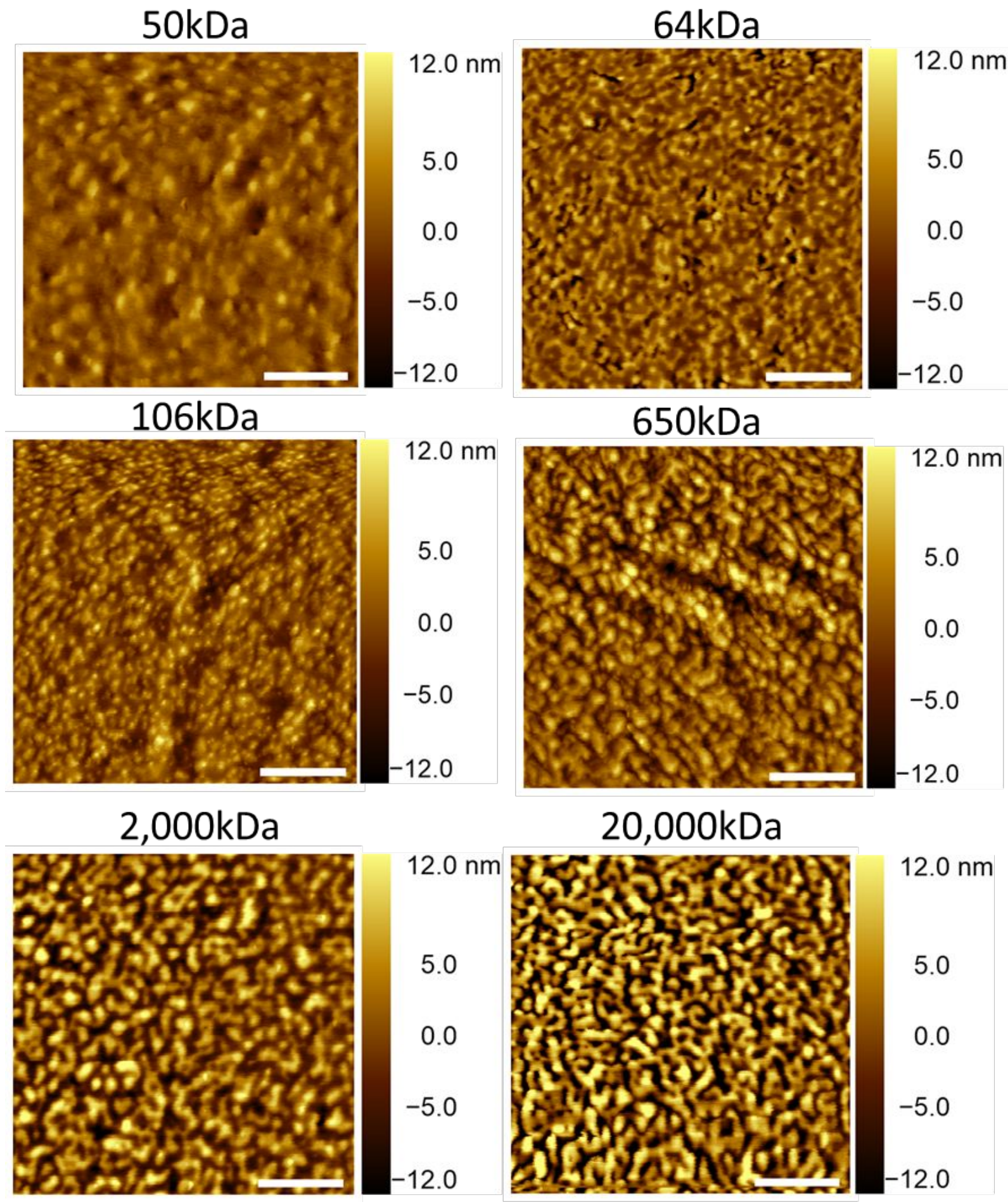

Figure S6: AFM images of the PS-Infiltrated NPG composites after infiltration at $150^{\circ} \mathrm{C}$ for 3 hours. The scale bars are $500 \mathrm{~nm}$. Color bar scales are kept constant to allow direct comparisons in film topology. 

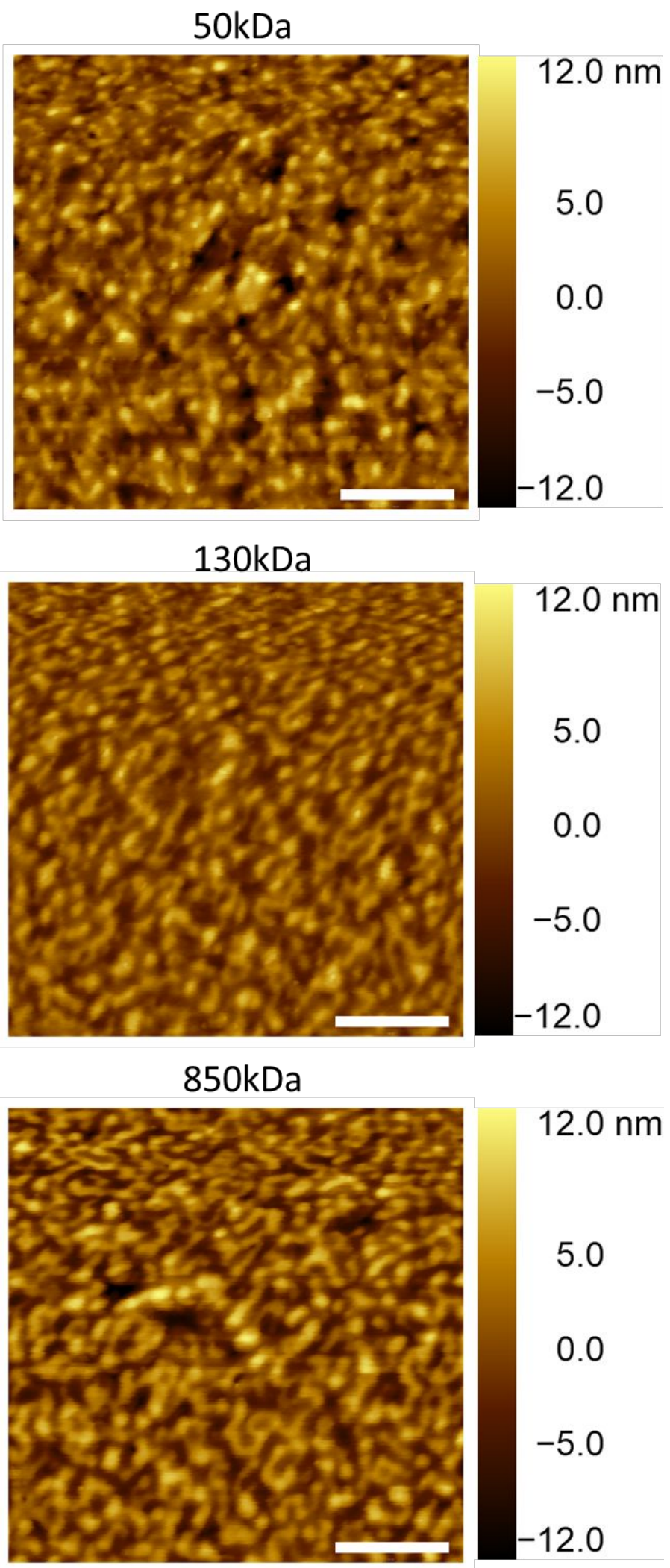

Figure S7: AFM images of the P2VP-Infiltrated NPG composites after infiltration at $150^{\circ} \mathrm{C}$ for 3 hours. The scale bars are $500 \mathrm{~nm}$. 


\section{Theoretical Prediction of Polymer Chain Diffusion}

AFM image analysis of the NPG composite films after infiltration is able to provide information on if the polymer chains have successfully reached the NPG film surface. Assuming the dynamics of the polymer chains are entirely diffusive in nature, then the distance $(l)$ a single chain can diffuse in time t are related by $l=\sqrt{\mathcal{D} t}$, where $\mathcal{D}$ is the diffusion coefficient of the polymer chain. Given that all the chain $M_{w}$ 's used in this study are well-entangled (i.e., $M_{w} \gg M_{e}$, the entanglement molecular weight) the diffusion of the chains should be governed by the reptation model. Following an analysis by Kramer et al, the diffusion coefficient of entangled PS chains is given as:

$$
\mathrm{D}=\frac{4}{15} \mathrm{M}_{\mathrm{e}} \mathrm{M}_{0} \mathrm{k}_{\mathrm{b}} \mathrm{TM}_{\mathrm{w}}{ }^{-2} \xi^{-1}
$$

Where $\mathrm{M}_{0}$ is the monomer molecular weight, $\mathrm{k}_{\mathrm{b}}$ is the Boltzmann constant, $\mathrm{T}$ is the temperature, and $\xi$ is the monomer friction coefficient measured from zero-shear rate viscosity experiments. We compare the diffusion distances predicted by reptation to our infiltration experiments by determining the diffusion coefficient necessary for a chain to diffuse $100 \mathrm{~nm}$ (i.e., the NPG film thickness, neglecting tortuosity effects) within a given infiltration time. We then use Eqn.1 to determine the maximum $\mathrm{M}_{\mathrm{w}}$ that would be able to diffuse this distance in the given time. For example, if the infiltration time is set at 3 hours, then the required diffusion coefficient for a chain to diffuse $100 \mathrm{~nm}$ is $\mathcal{D}={ }^{l^{2}} / t=9.25 \times 10^{-15} \mathrm{~cm}^{2} / \mathrm{sec}$, indicating that chains with $\mathrm{M}_{\mathrm{w}}<\approx 225 \mathrm{kDa}$ can diffuse at least $100 \mathrm{~nm}$ in the allotted time. We compare these predictions with our AFM roughness measurements for two different infiltration times in Figure S8 below. The shaded regions in each figure denote the chain molecular weights capable of diffusing $100 \mathrm{~nm}$ within the infiltration time given. In both cases, it appears that the reptation model under-predicts the diffusive motion of the polymer chains as chains with larger $\mathrm{M}_{\mathrm{w}}$ are able to fully infiltrate the NPG layer (e.g., PS chains with $\mathrm{M}_{\mathrm{w}}=650 \mathrm{kDa}$ fully infiltrate the NPG layer after 3 hours, while reptation theory predicts at best partial infiltration). This discrepancy is more clearly visible after 21 hours of annealing, where all chains are fully infiltrated regardless of $M_{w}$ while reptation theory predicts chains with $\mathrm{M}_{\mathrm{w}}<\approx 1,000 \mathrm{kDa}$ should not have reached full infiltration. This indicates that the dynamics of the infiltrated chains are significantly faster than that predicted by diffusion. 

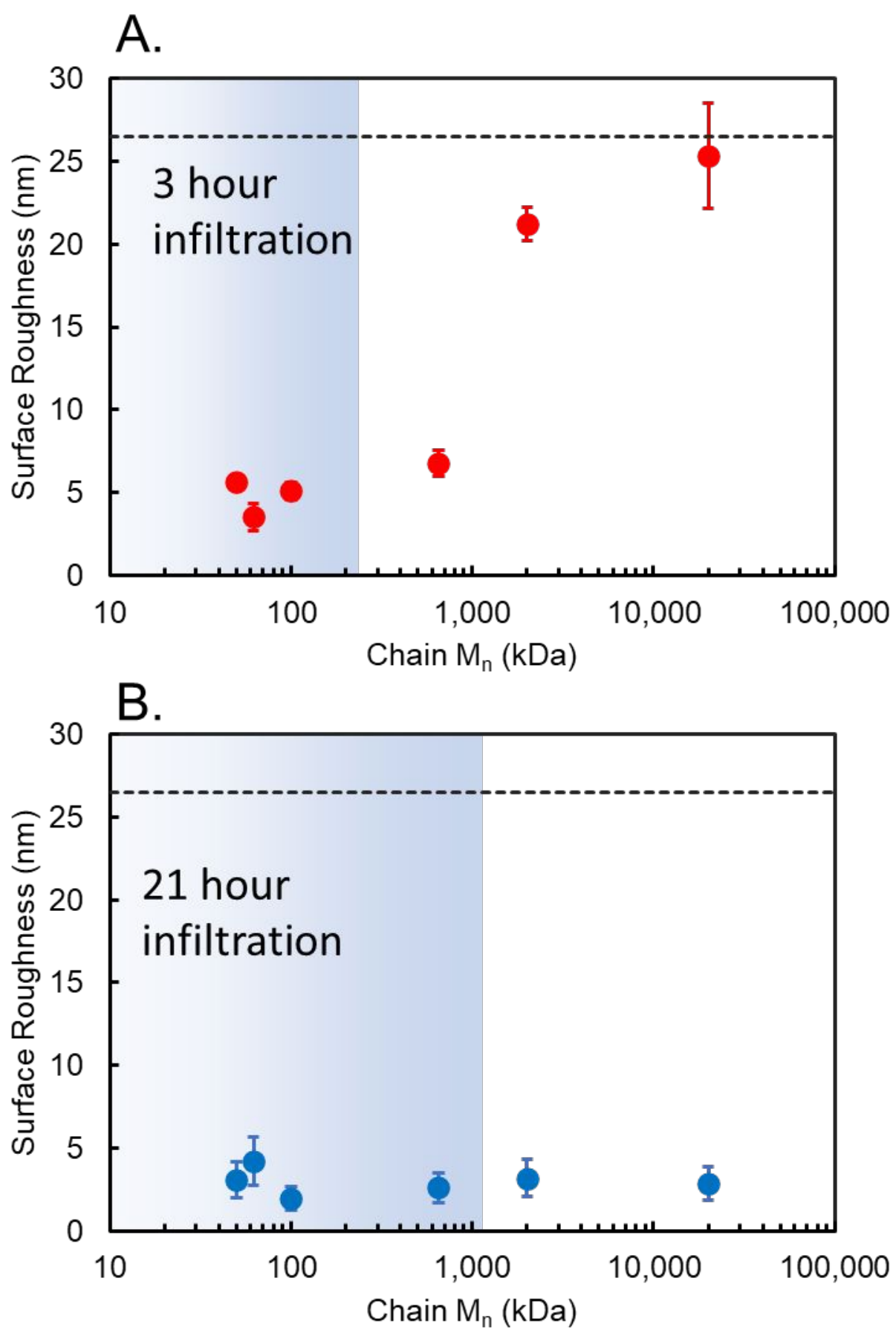

Figure S8: Comparison of AFM surface roughness data (indicating chain infiltration) after $3 \mathrm{hr}(\mathrm{A})$ and $21 \mathrm{hr}(B)$ of annealing as a function of chain $M_{w}$ with that required for a diffusion distance of $100 \mathrm{~nm}$ within the infiltration time based on reptation theory. Low surface roughness values $(<8$ $\mathrm{nm}$ ) are indicative of full infiltration. A portion of the experimental data with low surface roughness 
lie outside the region predicted by reptation theory, implying that the chain $\mathrm{M}_{\mathrm{w}}$ 's corresponding to these data points have diffused faster than that predicted by conventional theories.

\section{Ellipsometric Measurements and Modeling Procedure}

Spectroscopic ellipsometry of the NPG films was performed using an M2000 spectroscopic ellipsometer (J.A. Woollam) at 55, 60, 65, 70 and $75^{\circ}$ incident light angles. Data were collected on glass substrates with a frosted underside to minimize backscattering. Each acquisition was 10 seconds in length. Ellipsometry measures the ellipsometric angles $\Psi$ and $\Delta$ as a function of wavelength $(\lambda)$, which are related to the ratio of the reflection coefficients of the film by:

$$
\frac{\mathrm{r}_{\mathrm{p}}}{\mathrm{r}_{\mathrm{s}}}=\tan (\Psi) \mathrm{e}^{\mathrm{i} \Delta}
$$

Where $r_{p}$ and $r_{s}$ are the reflection coefficients for the $\mathrm{p}$ and $\mathrm{s}$ polarized light, respectively. The data are fit with various optical models to extract the complex refractive index of the film as a function of wavelength: $\tilde{n}=\mathrm{n}+\mathrm{ik}$; where $\mathrm{n}$ and $\mathrm{k}$ are the real and imaginary portions of the refractive index, respectively; equivalently, the fits can provide the real $\left(\varepsilon^{\prime}\right)$ and imaginary $\left(\varepsilon^{\prime \prime}\right)$ components of the dielectric constant. The best model exhibits the lowest mean squared error (MSE) using the fewest adjustable parameters. Representative ellipsometry spectra of the NPG films at a single incident angle $\left(70^{\circ}\right)$ is shown in Figure S9; representative spectra at all incident angles is shown in Figure S10A. The data is fit with various optical models to represent the film, with the goal to lower the mean squared error (MSE) between the data and the model using the smallest number of adjustable parameters.

A standard sample fitting procedure is described here for the as-cast NPG. The data is fit with a two-layer model; the first layer is the glass substrate, while the second is an effective medium of bulk (i.e., non-porous gold) and air, to represent the initially nanoporous material $\left(\tilde{n}=\tilde{n}_{A u} X_{A u}+\tilde{n}_{a i r}\right.$ $X_{\text {air }}$ ). In this context, the value of $X_{\text {air }}$ yields the porosity of the NPG film. For initial fitting, the porosity is set to be between $30-50 \%$ as determined from SAXS data, and the film thickness to be $100 \mathrm{~nm}$ as determined through SEM. Fitting the experimental data for NPG with bulk gold optical properties typically results in a MSE of $\approx 20-30$; an example of this fit is shown in Figure S8. For reference, a uniform, $100 \mathrm{~nm}$ film of pure polystyrene typically has MSE < 2 . Further simple changes to this model, i.e., by incorporating film surface roughness, refractive index gradients, etc., can further reduce the MSE, however there remain significant errors between the data and the model, and a number of the fit parameters that minimize error (i.e., a negative value for surface roughness) are non-physical. A summary of attempted models and their associated MSEs are shown in Table S1. The discrepancy between this effective medium model and the experimental results can be attributed to the unique 3-D microstructure of the gold, which is not captured in this simplistic model. 


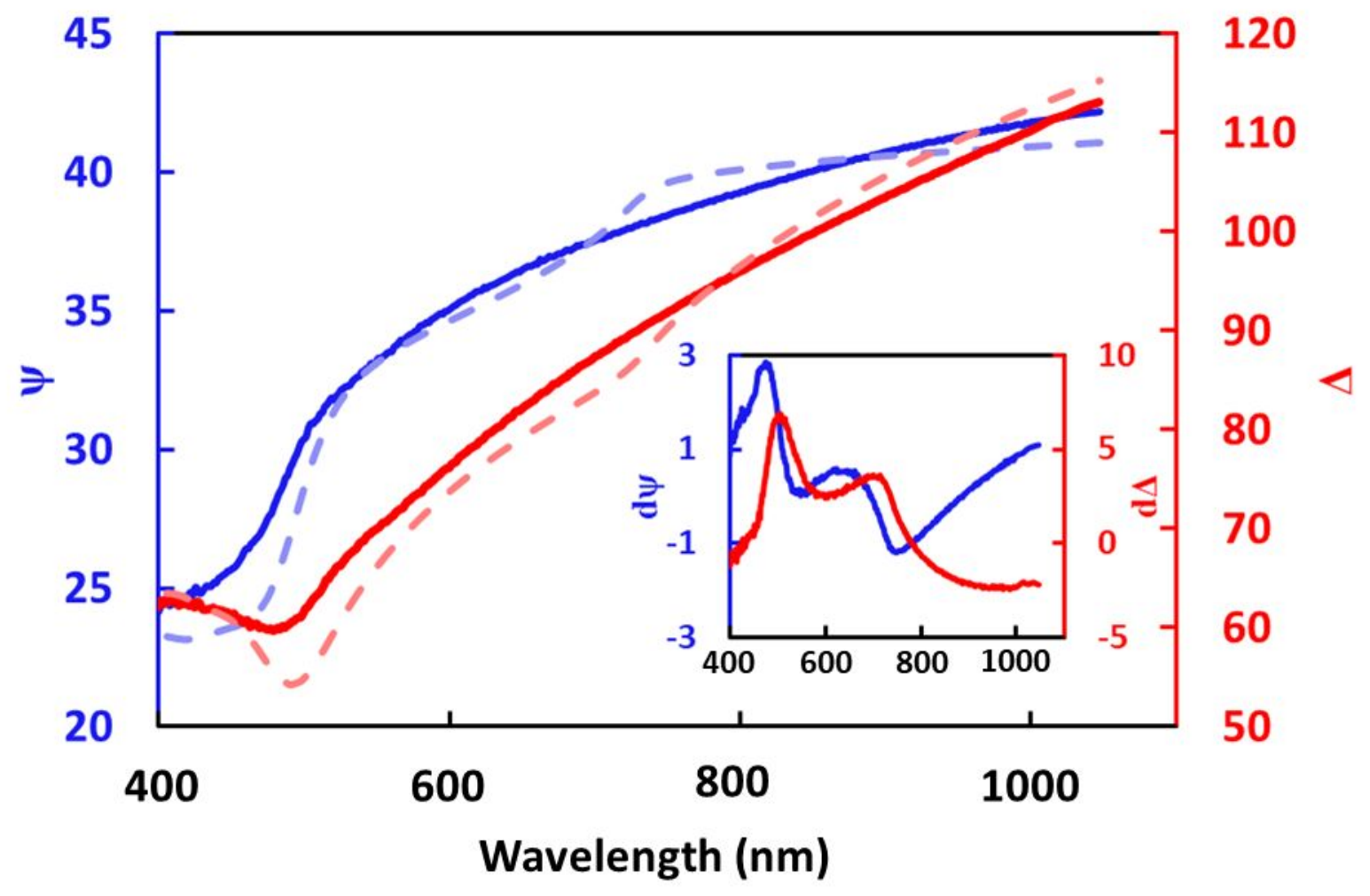

Figure S9: Spectroscopic ellipsometry data for as-cast NPG at a $70^{\circ}$ incident light angle. The dashed lines represent the best fit to the data assuming an effective medium composed of voids and bulk (i.e., non-porous) gold. Inset: relative errors between $\Psi$ and $\Delta$ using this model. The total MSE of this fit is $\approx 35$.

\begin{tabular}{|c|c|c|c|c|}
\hline Parameter & Ideal & Roughness & Grading & $\begin{array}{c}\text { Roughness \& } \\
\text { Grading }\end{array}$ \\
\hline MSE & 55.975 & 33.574 & 39.813 & 33.355 \\
\hline Roughness & N/A & $\begin{array}{c}-5.31 \pm 0.172 \\
\mathrm{~nm}\end{array}$ & $\mathrm{~N} / \mathrm{A}$ & $-6.26 \pm 0.822 \mathrm{~nm}$ \\
\hline \% Inhomogeneity & $\mathrm{N} / \mathrm{A}$ & $\mathrm{N} / \mathrm{A}$ & $60.40 \pm 2.994$ & $-12.62 \pm 10.487$ \\
\hline Thickness \# 1 & $\begin{array}{c}55.81 \pm \\
1.055 \mathrm{~nm}\end{array}$ & $\begin{array}{c}48.50 \pm 0.487 \\
\mathrm{~nm}\end{array}$ & $\begin{array}{c}60.61 \pm 0.885 \\
\mathrm{~nm}\end{array}$ & $46.69 \pm 1.585 \mathrm{~nm}$ \\
\hline Layer n @ 632.8 nm & 0.58764 & 0.58764 & 0.58764 & 0.58764 \\
\hline Layer k @ 632.8 nm & 1.5222 & 1.5222 & 1.5222 & 1.5222 \\
\hline
\end{tabular}

Table S1: Summary of various models usually employed to fit simple spectroscopic ellipsometry data-ideal (fits only A,B, and film thickness), added roughness, added index grading, and added added roughness and index grading. 
In the second model, the NPG layer was captured using a similar volumetric average, where $\tilde{\mathrm{n}}_{\mathrm{Au}}$ is instead fit to the experimental data using non-porous gold as a reference. Ellipsometry data in the wavelength range of $400 \mathrm{~nm}-1050 \mathrm{~nm}$ was used for fitting, with a total of 10 spline nodes. The optical properties of bulk gold (i.e., lacking any microstructure) was used as the starting point for the spline fitting. The basis-spline is constrained to be Kramers-Kronig consistent to obtain physically plausible relations between $\mathrm{n}$ and $\mathrm{k}$. A typical result of a basis-spline fit for the as-cast NPG is shown in Figure S10A; for clarity, only the model fit for an incident angle of $75^{\circ}$ is shown. This typically reduced the MSE to $\approx 4$. $\tilde{n}$ is then converted into the complex dielectric function of the material $\tilde{\varepsilon}$ using the relation $\tilde{\varepsilon}=\tilde{n}^{2}$.

We focus first on the refractive index characteristics in the pure NPG films; $n$ and $k$ of as-cast NPG as a function of wavelength is shown in Figure S10B - data for $n$ and $\mathrm{k}$ of non-porous gold used in the volumetric average fit is shown for comparison. The most striking difference between the two data sets is the presence of a single, broad, maximum near $800 \mathrm{~nm}$ in the imaginary refractive index of the as-cast NPG; this absorption feature is noticeably absent in the optical properties of the non-porous. This appears to be the primary reason why modeling the raw ellipsometry data (Figure S9) using a volumetric average fails to accurately capture the trends in the data as a function of wavelength. However, analysis of ñ alone cannot explain the emergence of this observed change.

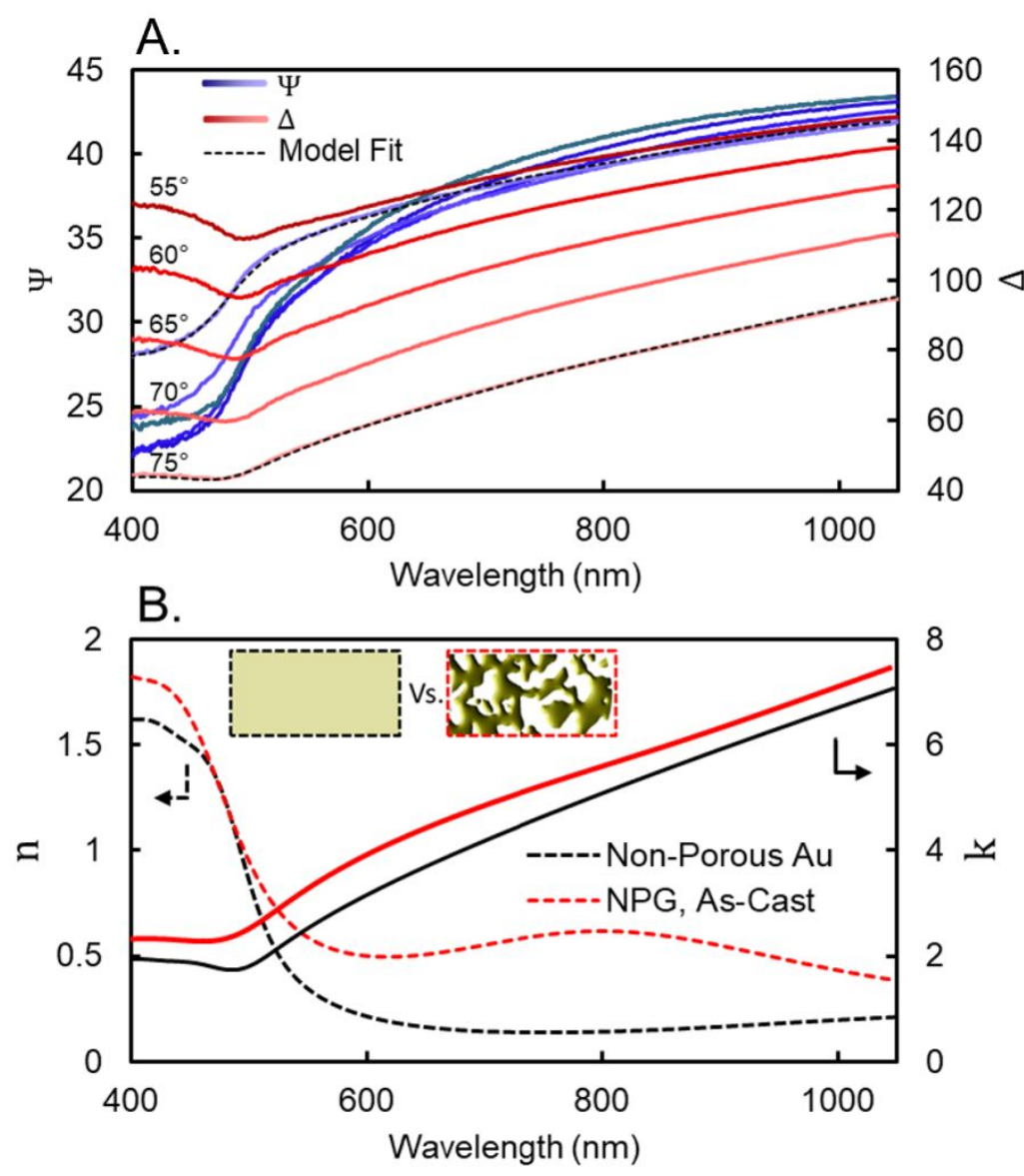

Figure S10: (A) Raw ellipsometric data of an as-cast NPG film as a function of wavelength at five incident light angles. The incident angle increases with increasing color brightness. For clarity, 
only the model fit at $75^{\circ}$ incident light angle is shown. (B) Comparison of the real ( $n$, dashed lines) and imaginary ( $k$, solid lines) portions of the refractive index of non-porous Au and as-cast NPG films. Inset schematics show a structural comparison between the volumetric average model (black lines) and the nano-porous model (red lines).

We instead consider the dielectric loss function $\varepsilon$ ", which can be modeled using a series of harmonic oscillators to assign structural origins to these optical properties. This was performed by first modeling the optical response of bulk gold. We fit the pure gold data to a total of three distinct oscillators. The first two are a pair of Gaussian oscillators that are used to properly model the low-wavelength data; these do not significantly contribute to the fit above $\approx 600 \mathrm{~nm}$, in the range of the adsorption feature seen in the experimental data. The third oscillator is a Drude model to describe the motion of unbounded electrons, described by the relation:

$$
\varepsilon^{\prime \prime}=\frac{\omega_{\mathrm{p}}^{2} \Gamma}{\omega\left(\omega^{2}+\Gamma^{2}\right)}
$$

Where $\omega_{p}$ is the plasma frequency and $\Gamma$ is the collision frequency of the oscillator. The fit parameters for pure gold are shown in Figure S11A. The optical properties of non-porous gold are shown as a solid black line in Figure S11B below; the grey dashed lines represent the contributions from the three harmonic oscillator models. These three oscillators adequately capture the optical properties of gold and are similar to past modeling results on gold nanorod materials. ${ }^{1}$ However, this model alone does not model the dielectric loss of the NPG materials, particularly the non-monotonic feature in the vicinity of ca. $750 \mathrm{~nm}$. We therefore add a Lorentzian oscillator to our model fit (without changing the fit parameters of the bulk gold oscillators by more than $5 \%$ to improve goodness-of-fit), due to the observed broadness of the non-monotonic feature:

$$
\tilde{\varepsilon}=\frac{A m p \times B r \times E_{0}}{E_{0}^{2}-E^{2}-i \times B r \times E}
$$




\begin{tabular}{|c|c|c|c|c|c|}
\hline Oscillator & Amplitude & Breadth & $\begin{array}{l}E_{0} \\
(e V)\end{array}$ & $\begin{array}{l}\text { Resistivity (Ohm } \\
\text { cm) }\end{array}$ & $\begin{array}{c}\text { Scattering time, } \\
1 / \Gamma(\mathrm{fs})\end{array}$ \\
\hline \multirow{3}{*}{$\begin{array}{c}\text { Gaussian \#1 } \\
\text { Gaussian \#2 } \\
\text { Drude }\end{array}$} & 1.612 & 0.3263 & 2.687 & N/A & N/A \\
\hline & 9.674 & 1.800 & 3.777 & N/A & $\mathrm{N} / \mathrm{A}$ \\
\hline & N/A & N/A & N/A & $3.14 \times 10^{-6}$ & 9.926 \\
\hline
\end{tabular}

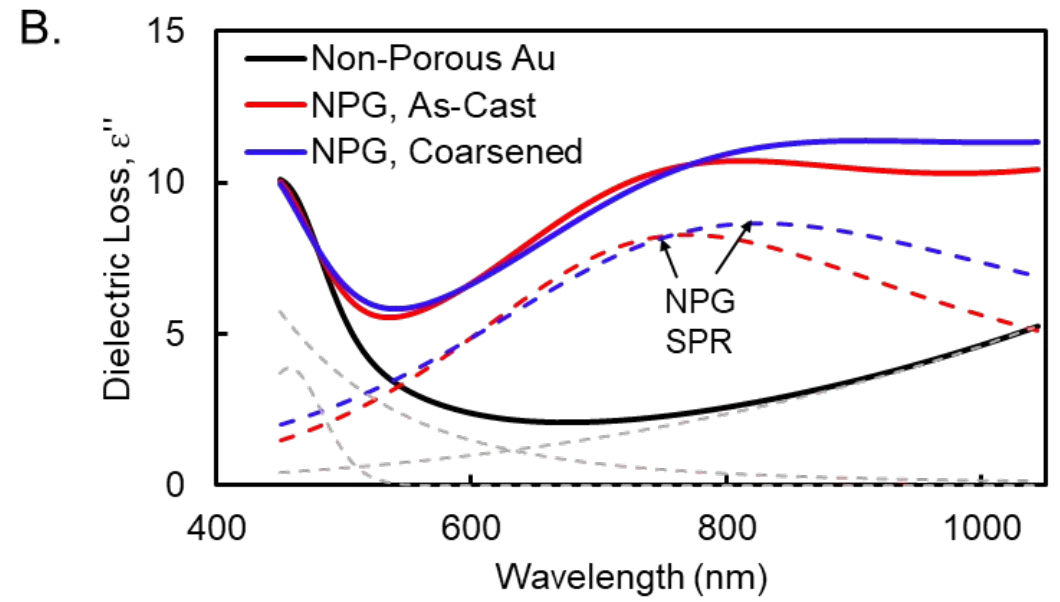

Figure S11: (A) Oscillator parameters for modeling of bulk gold. These parameters are used to fit all NPG data-sets. (B) Dielectric loss of NPG materials based on optical modeling of the ellipsometry data. Solid lines denote the complete dielectric loss, while dashed lines represent the individual harmonic oscillators obtained from fitting the data. The colored dashed lines are Lorentzian oscillator components of the dielectric loss of the NPG materials, which we attribute to surface plasmon resonance. Fits to non-porous gold (shown as grey dashed lines) do not include this component.

Where $\mathrm{E}$ is the photon energy, Amp and $\mathrm{Br}$ are the amplitude and breadth of the distribution, respectively, and $E_{0}$ is the center energy. This dual-feature model is sufficient to model the dielectric properties of the NPG thin film. The added Lorentzian fit for as-cast NPG is shown as a red dashed line in Figure S10B; the full dielectric loss of the NPG is obtained by combining this function with the three oscillators representing non-porous gold.

We attribute this additional feature to a surface plasmon resonance (SPR) that manifests as a result of the ligament structure present in the NPG material, but absent in non-porous Au. The fit $\mathrm{E}_{0}$ value (typically in the range of $1.50-1.65 \mathrm{eV}$ ) is used to compute the wavelength of the SPR, which is related to the average length of the NPG ligaments (measured as $75 \pm 10 \mathrm{~nm}$ from analysis of AFM, SEM and SAXS data). This dispersity in ligament length explains the broadness of the SPR feature. Past experiments probing the optical properties of NPG materials using UVVisible spectroscopy ${ }^{2-4}$ reveal a similar plasmon resonance feature which corresponds to the average ligament length (though at slightly lower wavelengths), as well as a weak transverse plasmon resonance near ca. $550 \mathrm{~nm}$, attributed to the NPG ligament width; while we do not directly observe this feature, this range is comparable to that of the Gaussian oscillators we have used to model the optical response of non-porous $A u$ and may therefore not be able to be modeled.

Upon annealing at $175^{\circ} \mathrm{C}$ for 3 hours, the SPR shifts to a longer wavelength by approximately 45 $\mathrm{nm}$ upon coarsening (blue lines in Figure S11B). This shift in SPR indicates that ellipsometry is 
sensitive to small changes in the local structure of the gold ligament network. The ellipsometric modeling is also able to measure the porosity (i.e., the volume fraction of air in the material) as $40 \pm 5 \%$, which is consistent with the expected value based on the original metal alloy pre-cursor $\left(\mathrm{Au}_{65} \mathrm{Ag}_{35}\right.$ atomic \%). In-situ ellipsometry measurements of the NPG film conducted during the coarsening process also shows that the SPR peak wavelength is relatively constant after 1 hour, indicating a stable film microstructure. These data are also in good agreement with ex-situ measurements of the SPR wavelength taken after progressively longer annealing times as shown in Figure S12.

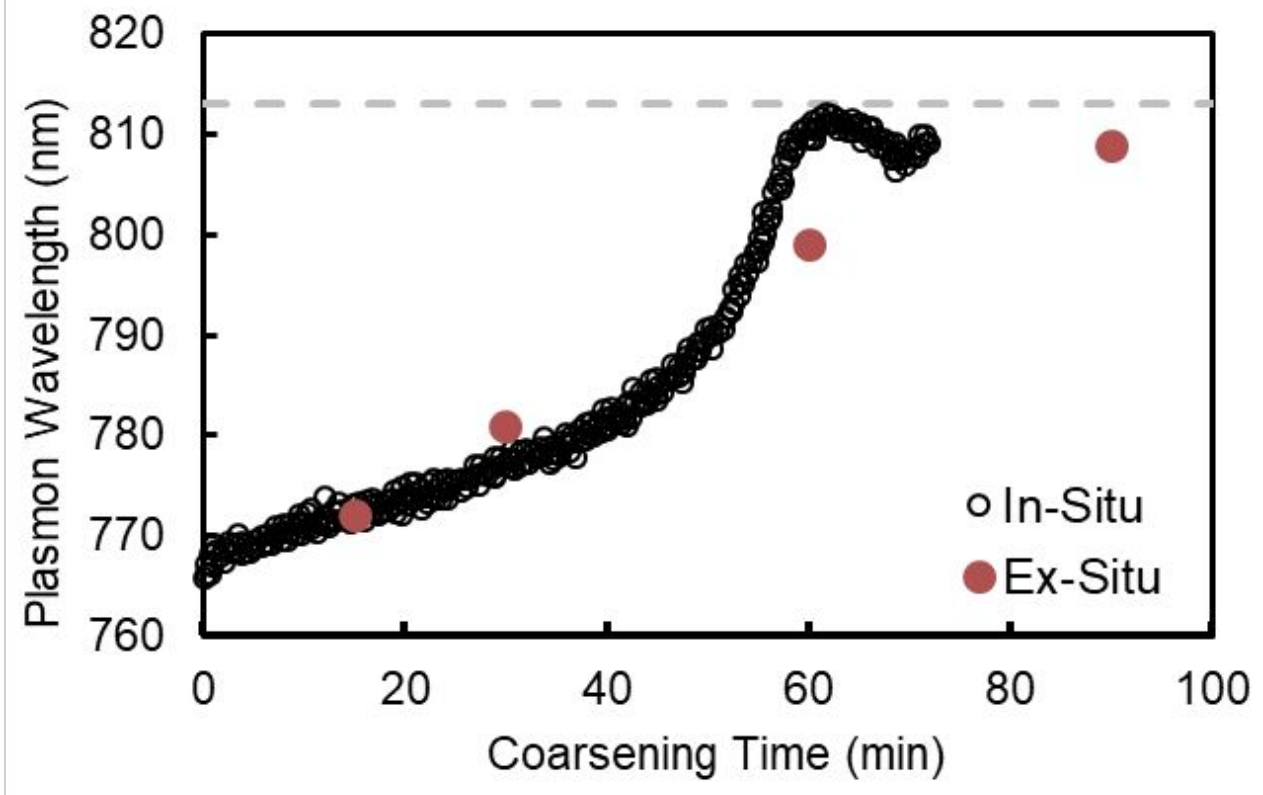

Figure S12: In-situ spectroscopic ellipsometry modeling of the NPG plasmon wavelength on thermal coarsening. The Lorentzian oscillator parameters are the only fit parameters allowed to change. The bulk gold oscillator parameters are measured at $150^{\circ} \mathrm{C}$ and are fixed during this modeling procedure.

Measurements of the polymer-infiltrated NPG used the fully-modeled NPG material as a basis. However, the composite is instead modeled as an effective medium of NPG and polymer. The polymer portion was modeled using the Cauchy dispersion relation:

$$
\mathrm{n}=\mathrm{A}+\frac{\mathrm{B}}{\lambda^{2}} \quad, \mathrm{k}=0
$$

Both $A$ and $B$ were restricted to positive values to obtain physically realistic data. The assumption that the polymer portion of the model can be fit independently of the NPG is based off the expectation that the substitution of the void spaces for polymer should not affect the local dielectric functions that arise from the NPG microstructure. To test this hypothesis, we allow the oscillator parameters to vary and re-fit the data. However, there is no significant change in the modeled dielectric loss of the NPG in both PS- and P2VP-infiltrated materials, as seen in Figure S13. 


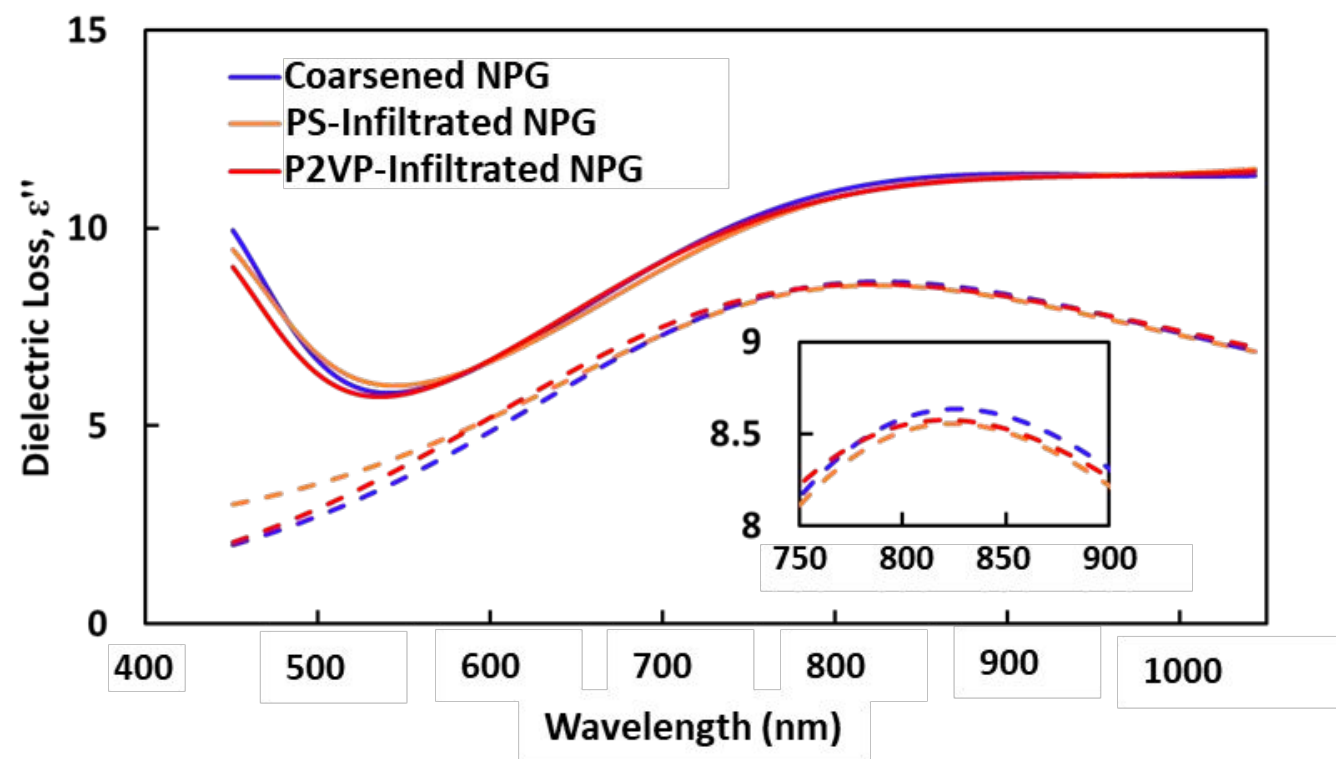

Figure S13: Comparison of the dielectric loss of the NPG composites after infiltrating with PS-64 or P2VP-50. Inset: the fit plasmon resonance zoomed in near the peak maximum. The dashed lines represent the contribution of the NPG surface plasmon resonance to the dielectric loss.

Optical modeling of the aged NPG materials was performed in a similar fashion; however, the initial model begins with an effective medium of the coarsened NPG film (described through a series of oscillators as discussed above) and voids; this model typically produced an MSE $\approx 12$. For this data, the oscillator parameters are initially fixed and the porosity of the film varied. This reduced the MSE to $\approx 7$. Allowing the Lorentzian oscillator parameters to also vary further reduced the MSE to $\approx 3$. The $\mathrm{n}$ and $\mathrm{k}$ of the aged NPG layer as a function of wavelength is shown in Figure S14. 


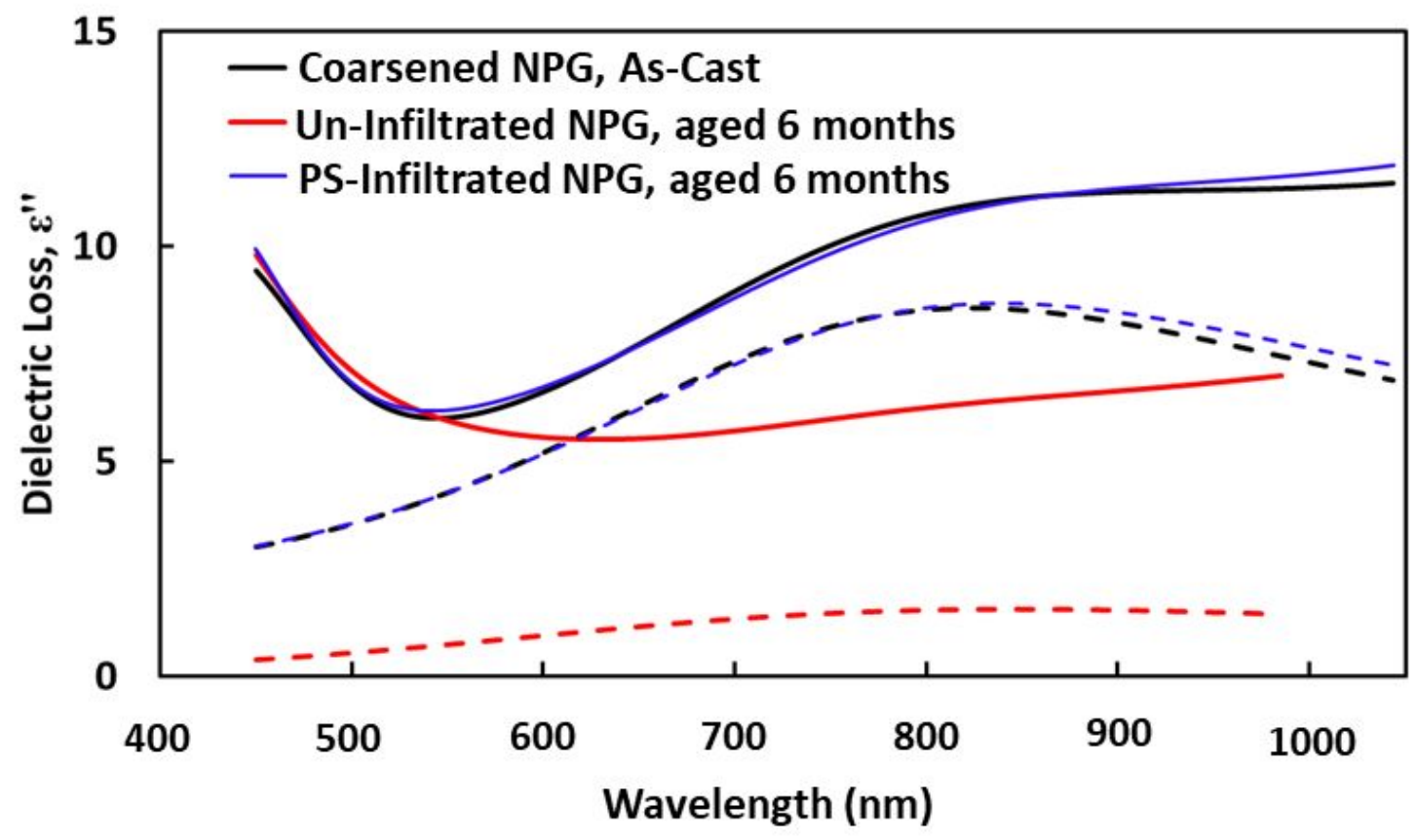

Figure S14: Comparison of the dielectric loss of the NPG composites after temporal aging for 6 months. The dielectric loss and plasmon resonance of the as-cast NPG is shown as a reference. The dashed lines represent the contribution of the NPG surface plasmon resonance to the dielectric loss.

\section{In-situ Infiltration and Glass Transition Temperature Measurements}

In-situ infiltration and glass transition temperature measurements are performed at a single incidence angle of $70^{\circ}$; the acquisition time is approximately 1 second. The sample temperature is controlled using a Linkam THMSEL350 temperature-controlled stage (Linkam, UK). Stacked bilayers of polymer and NPG were fabricated by floating the NPG flim off of a glass substrate before depositing it on top of a spin-cast polymer film of the desired thickness (typically 100nm) For infiltration experiments, a stacked bilayer of polymer and NPG was heated to $150^{\circ} \mathrm{C}$ and held at that temperature for 3 hours under vacuum. The films were then allowed to cool to room temperature. The resulting data were fit with a two-layer model; the first layer represents the glass substrate, while the second layer represents the NPG film. However, in these experiments the refractive index of the pores was fit as a function of time/temperature in order to capture the infiltration kinetics. The total fill fraction was then computed using the relation:

$$
\text { Fill Fraction }=\frac{n_{p}(t)-n_{p, 0}}{n_{p o l y}-n_{p, 0}}
$$

Where $\mathrm{n}_{\mathrm{p}}(\mathrm{t})$ is the pores' refractive index as a function of time, $\mathrm{n}_{\mathrm{p}, 0}$ is the pores initial refractive index $(\approx 1)$, and $\mathrm{n}_{\text {poly }}$ is the refractive index of the polymer undergoing infiltration. We note that this model does not include the optical properties of the polymer under-layer before or during the infiltration process; we find that the polymer contributes weakly to the overall optical properties of 
the stacked bi-layer, likely due to the greater optical activity of the NPG film. We also find that arbitrarily attempting to model the data for a pure NPG with a thickness $>100 \mathrm{~nm}$ does not affect the fit MSE, indicating that beyond this limit, we are insensitive to the film optics (again likely due to the complex optical response of the NPG).

Glass transition temperature measurements were performed by first heating the infiltrated NPG/polymer composite to $165^{\circ} \mathrm{C}$ for 10 minutes. The composite was then cooled to room temperature at a rate of $10^{\circ} \mathrm{C} / \mathrm{min}$. The data was modeled in a similar fashion as the in-situ infiltration experiments. The $\mathrm{T}_{\mathrm{g}}$ of the infiltrated polymer was determined as the intersection of the linear fits of the glass and supercooled liquid regions in the plot of $n_{p}$ versus $T$. The $\mathrm{T}_{\mathrm{g}}{ }^{+}$and $\mathrm{T}_{\mathrm{g}}{ }^{-}$ values were obtained by fitting the polymers refractive index as a function of temperature, using the equation ${ }^{5}$ :

$$
\mathrm{n}_{\mathrm{p}}(\mathrm{T})=\mathrm{w}\left(\frac{\mathrm{S}-\mathrm{G}}{2}\right) \ln \left(\cosh \left(\frac{\mathrm{T}-\mathrm{T}_{\mathrm{g}}}{\mathrm{w}}\right)\right)+\left(\mathrm{T}-\mathrm{T}_{\mathrm{g}}\right)\left(\frac{\mathrm{S}+\mathrm{G}}{2}\right)+\mathrm{c}
$$

Where $\mathrm{S}$ and $\mathrm{G}$ are the slopes of the supercooled liquid and glassy regimes, $\mathrm{w}$ is the breadth of the glass transition, and $\mathrm{c}$ is the refractive index at $\mathrm{T}_{\mathrm{g}}$. The high and low onsets of $\mathrm{T}_{\mathrm{g}}$ (i.e., $\mathrm{T}_{\mathrm{g}}{ }^{+}$and $\mathrm{T}_{\mathrm{g}}{ }^{-}$) were obtained by assuming $\mathrm{T}_{\mathrm{g}}{ }^{ \pm}=\mathrm{T}_{\mathrm{g}} \pm \mathrm{w} / 2$. A representative fit for a thin film $(\approx 45 \mathrm{~nm})$ of PS-62 is shown in Figure S15.

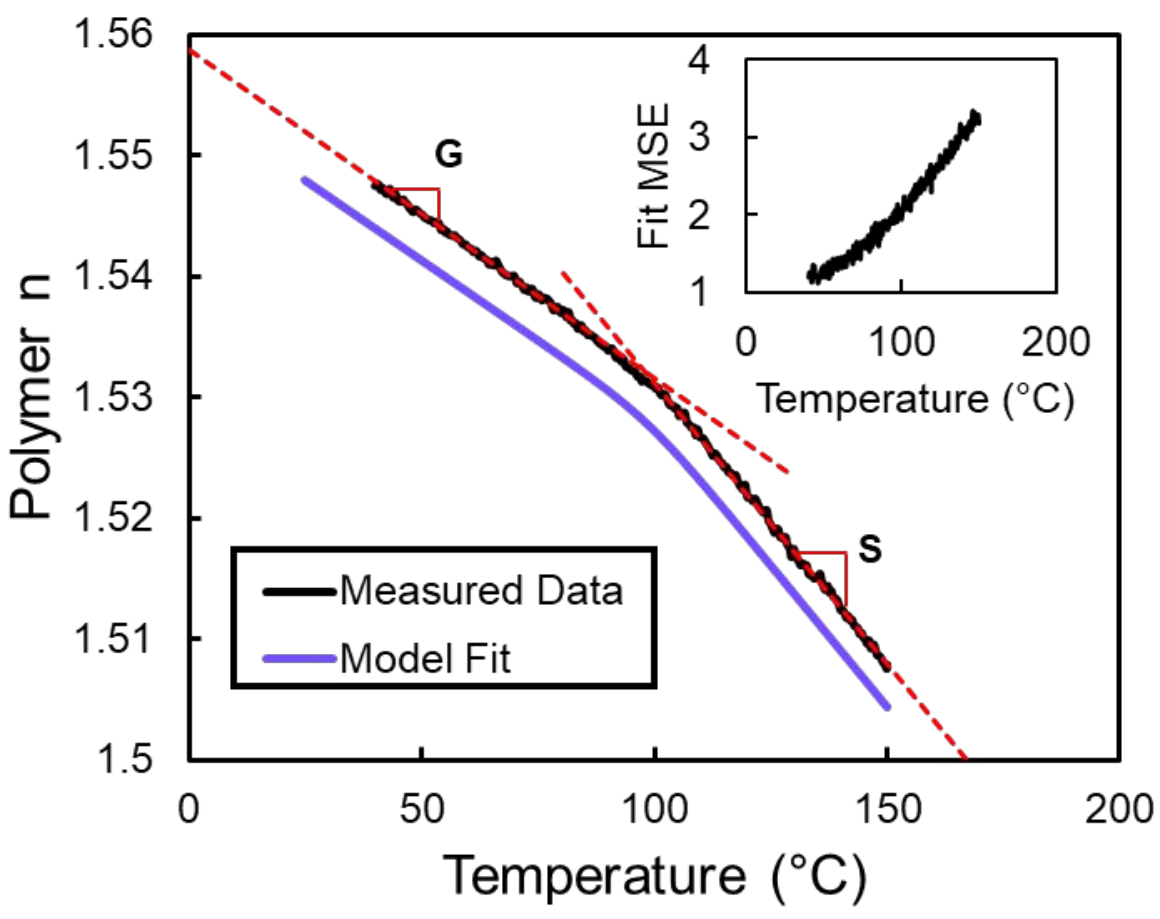

Figure S15: Representative fitting of $T_{g}, T_{g}{ }^{+}$and $T_{g}{ }^{-}$from $n(T)$ curves. The red dashed lines are fits to the linear portions of the data and represent the slopes of the supercooled liquid (S) and glassy regimes $(\mathrm{G})$. The model fit from Equation 7 is shown as a solid blue line and is shifted vertically for clarity. Inset: fit MSE of the data. 


\section{Supporting Images and Data}
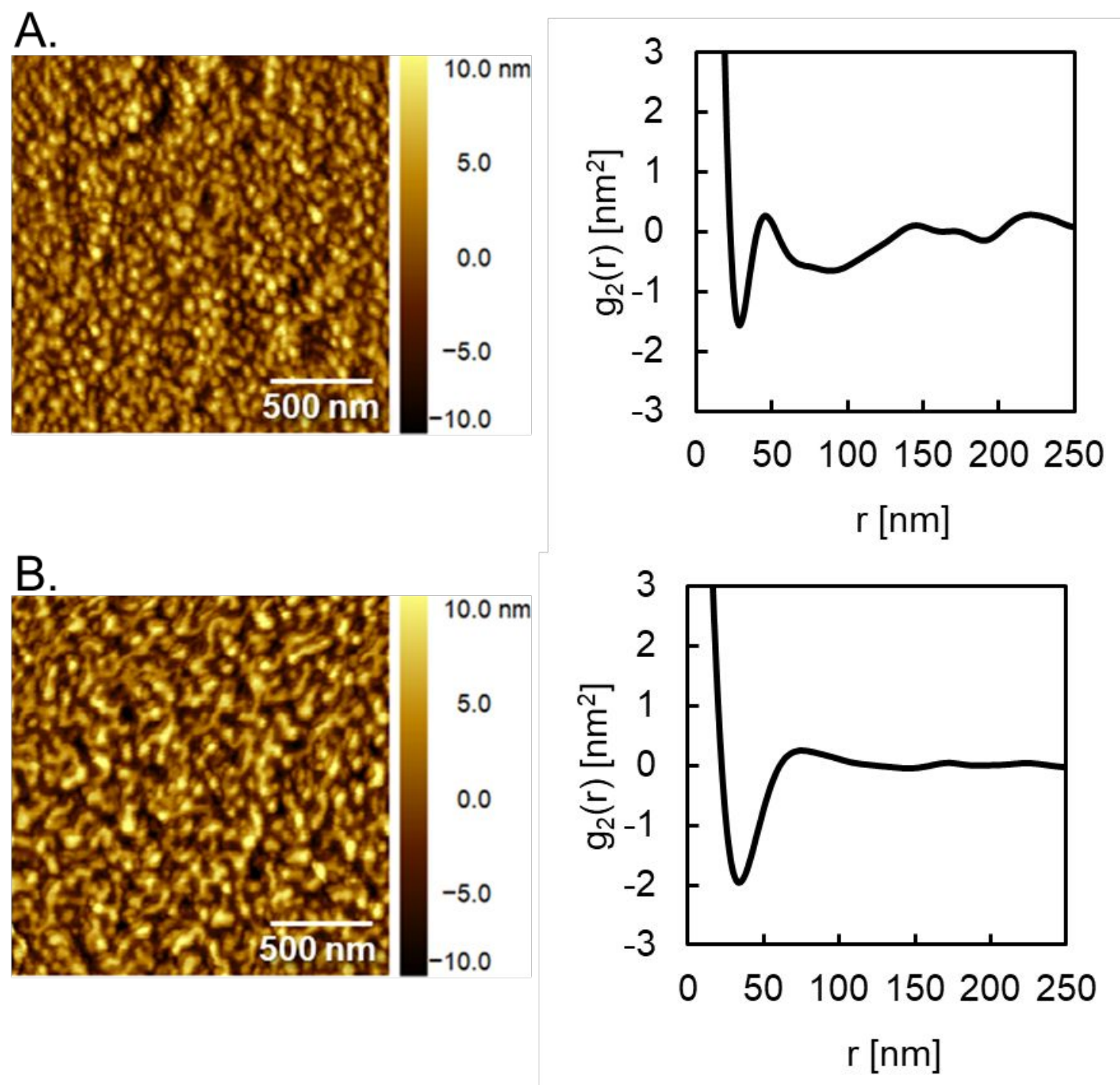

Figure S16: AFM height image and radial auto-correlation function for (A) as-cast NPG and (B) NPG after thermal coarsening at $175^{\circ} \mathrm{C}$ for 3 hours. The first peak in the radial auto-correlation function represents the characteristic dimension of the NPG ligaments and is compared with that extracted from SAXS profiles. 

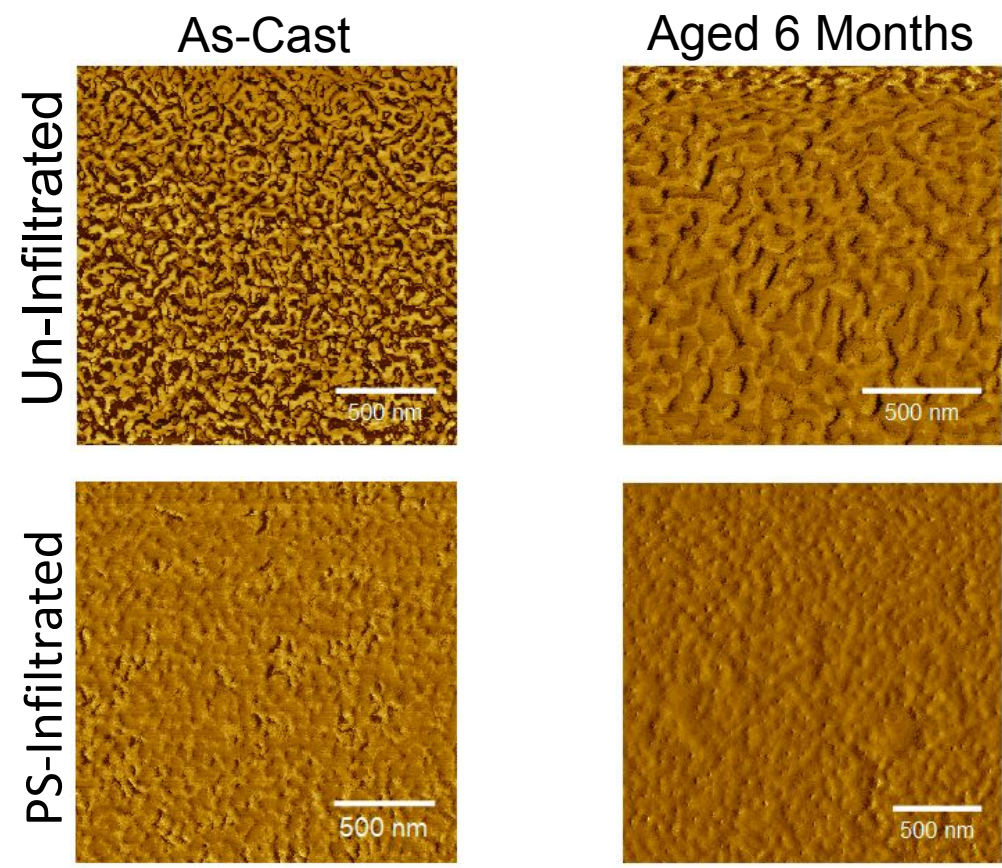

Figure S17: AFM images of non-infiltrated and PS-infiltrated NPG as-prepared versus aged at ambient conditions for 6 months. Image scale bars are $500 \mathrm{~nm}$.

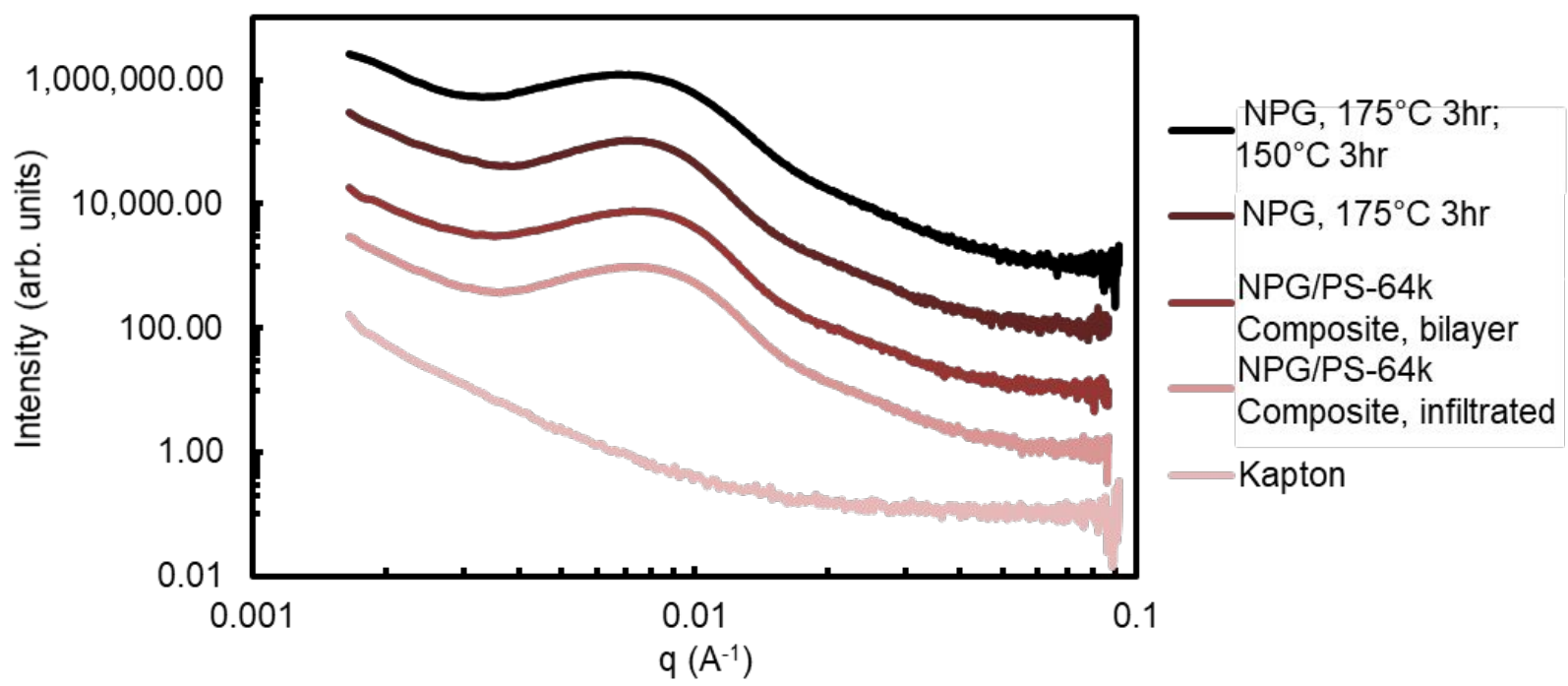

Figure S18: 1-Dimensional SAXS profiles of the NPG films and composites after the thermal processing techniques used in this study. 


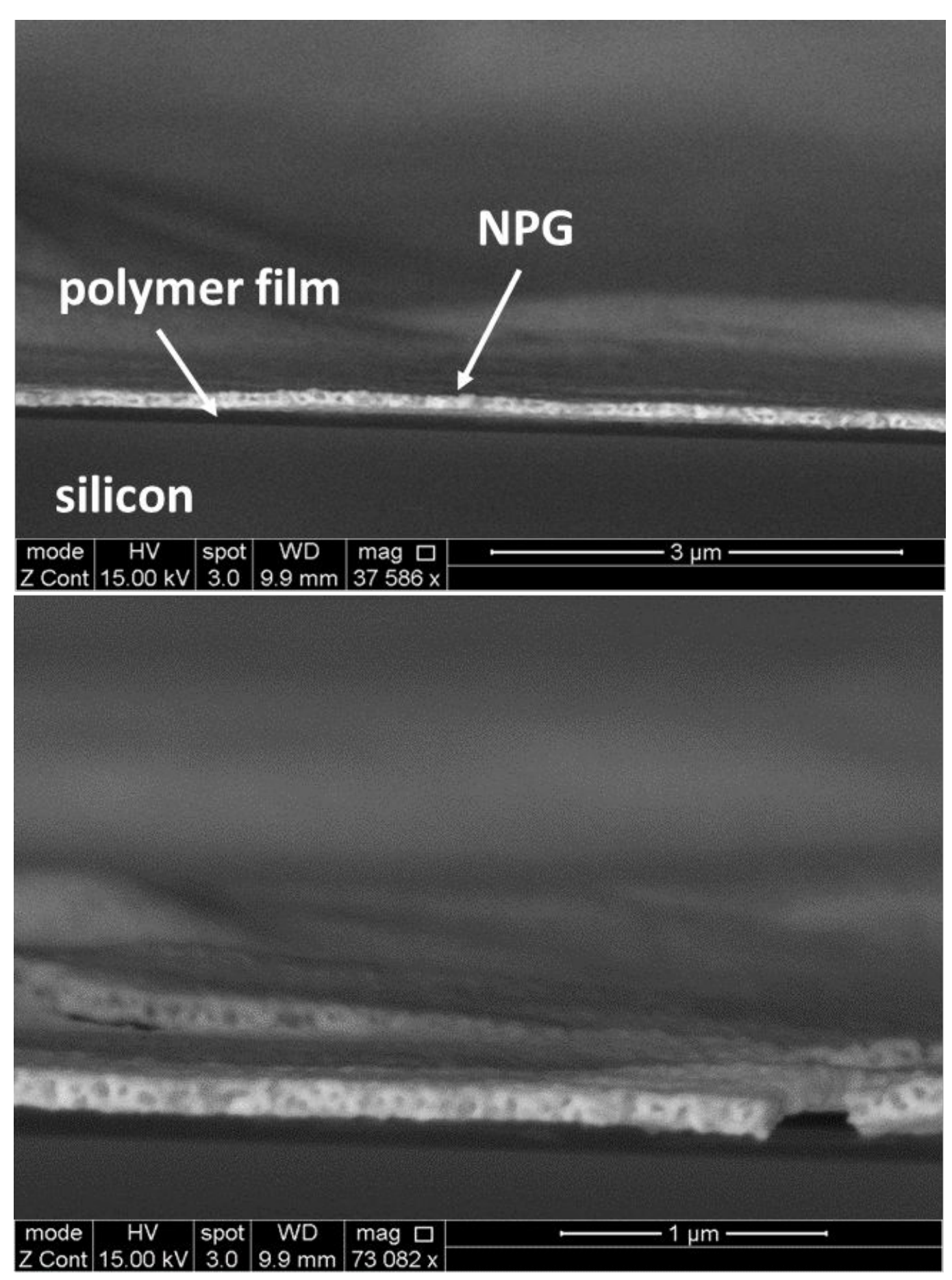

Figure S89: Additional SEM images of NPG/PS-64 stacked bilayers 


\section{Infiltrated NPG}
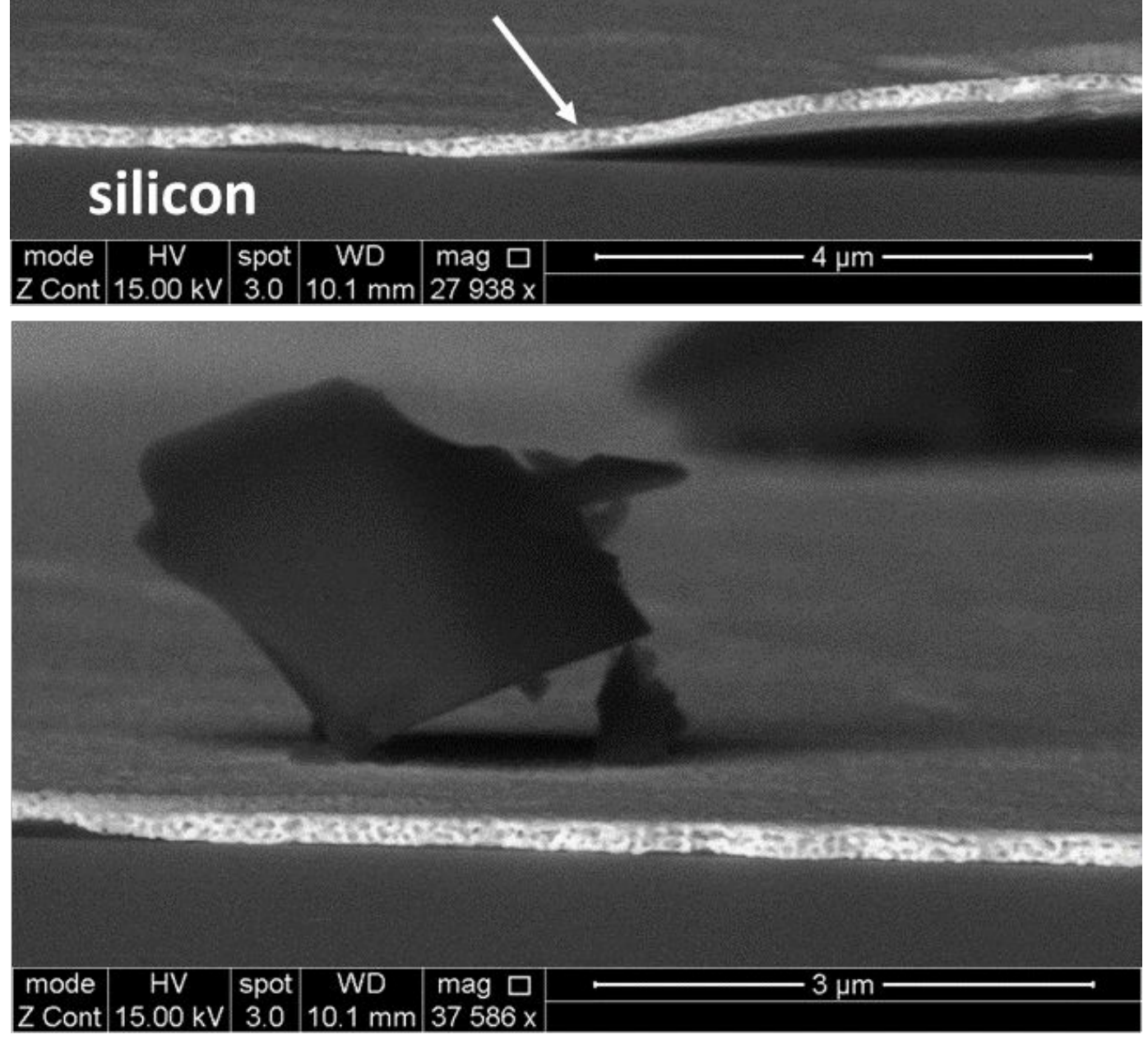

Figure S20: Additional SEM images of NPG/PS-64 composites after thermal infiltration at $150^{\circ} \mathrm{C}$ for 3 hours 


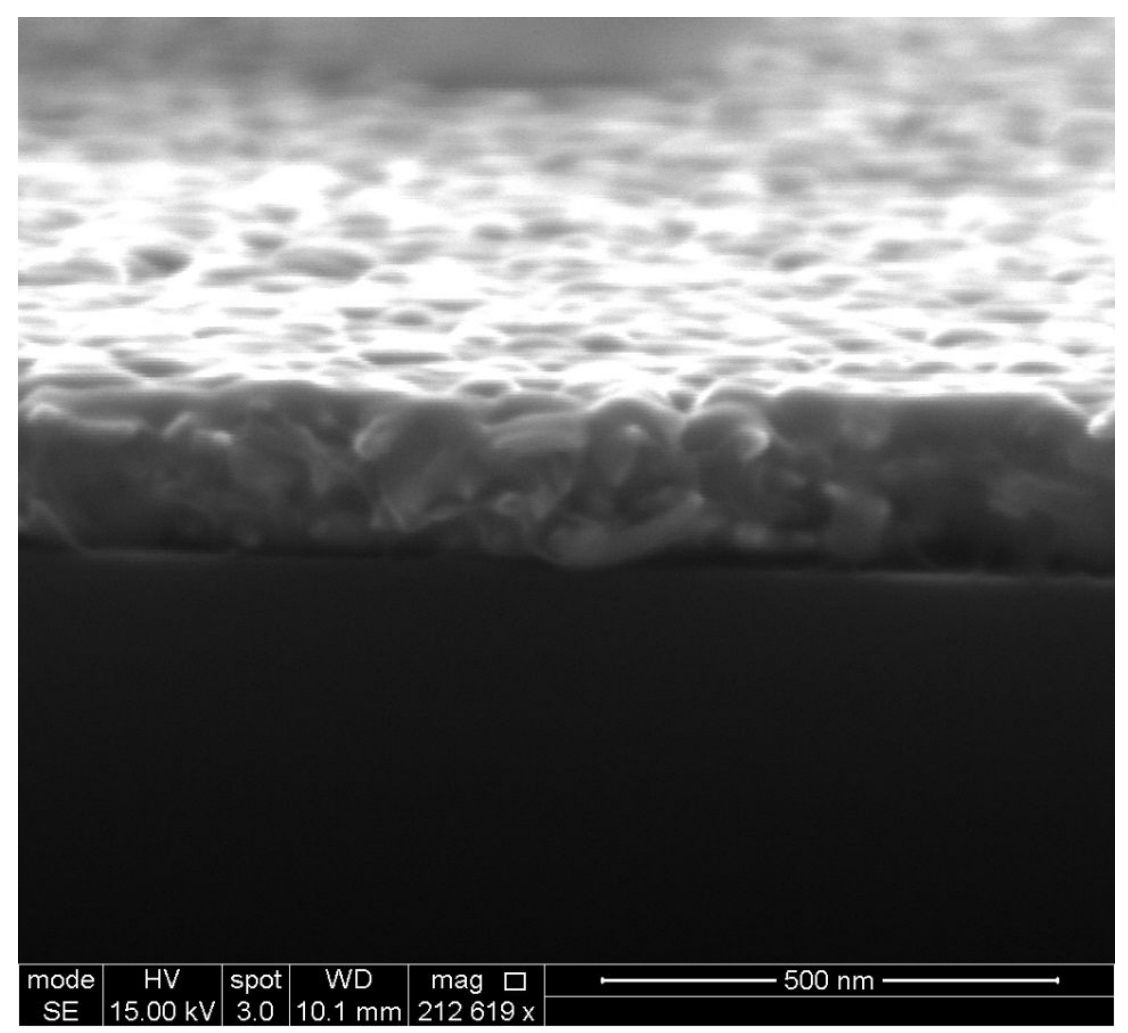

Figure S21: Cross-sectional SEM image of NPG/PS-64 composite after thermal infiltration at $150^{\circ} \mathrm{C}$ for 3 hours.

A.

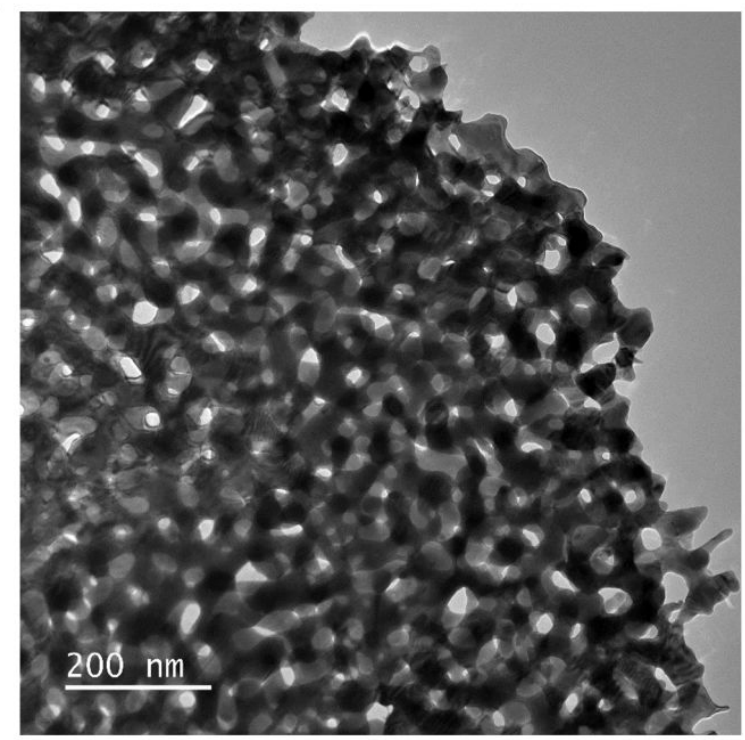

B.

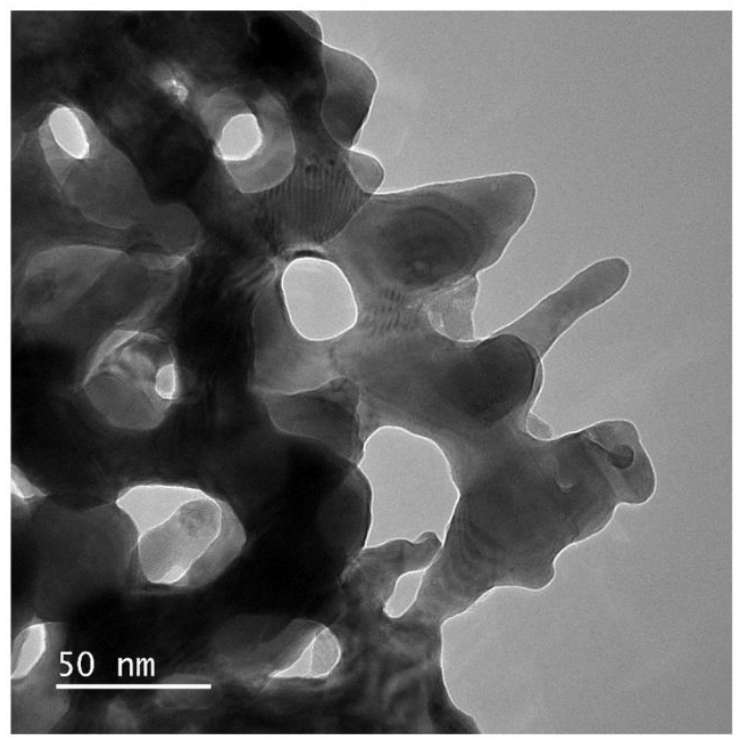

Figure S22: TEM images of as-cast NPG films at (A) low and (B) high magnification. 


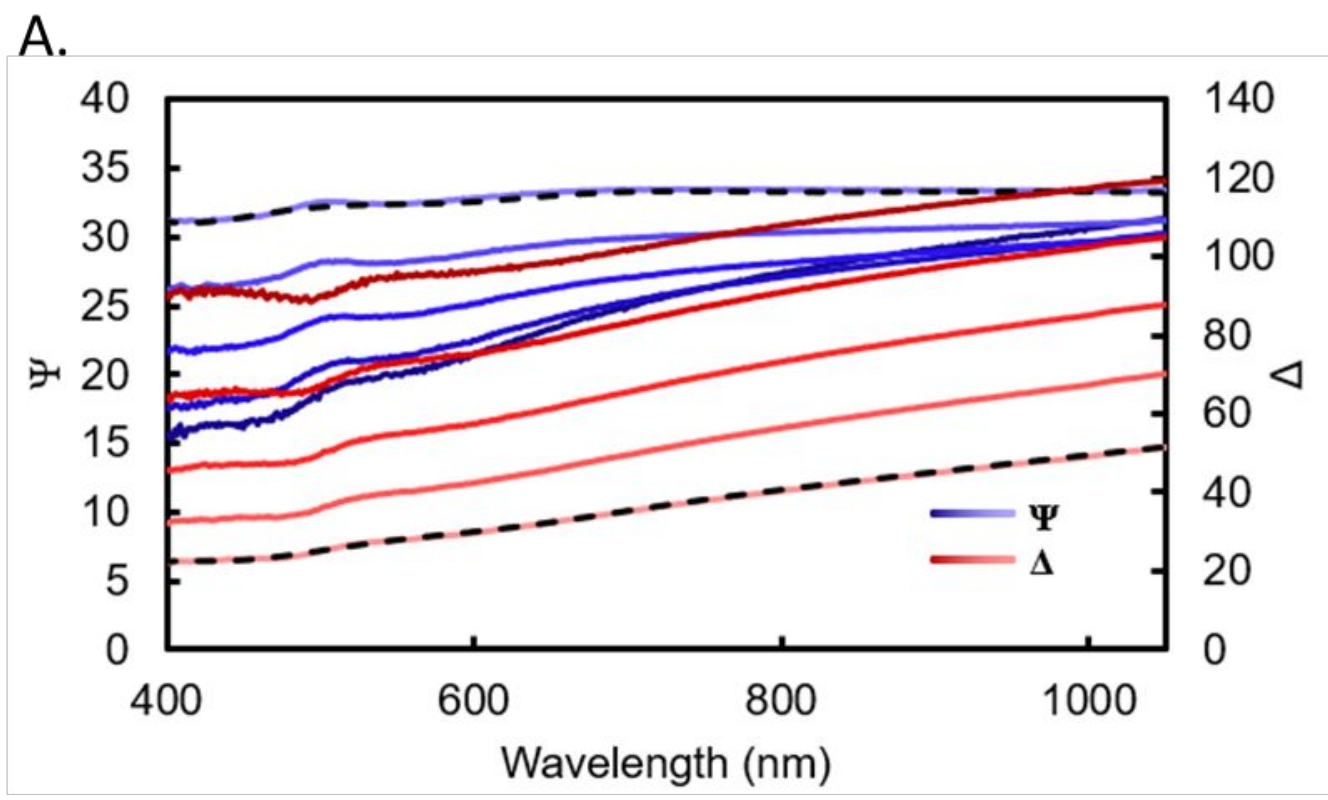

B.

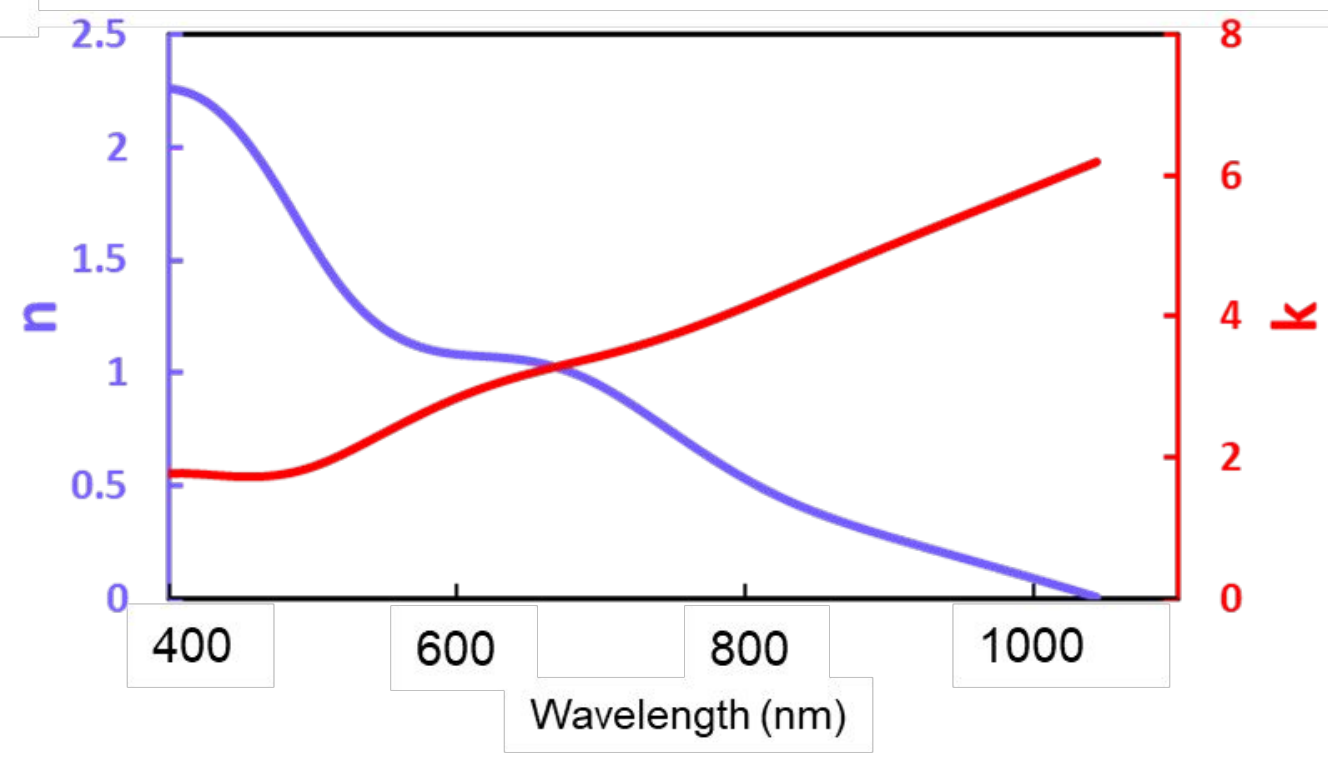

Figure S23: (A) raw ellipsometric data and (B) modeled complex refractive index of NPG fabricated from $\mathrm{Au}_{25} \mathrm{Ag}_{75}$. The modeled porosity of this material is $66 \%$. For clarity, only the model fit at $75^{\circ}$ incident light angle is shown. 


\section{References}

1. Glor, E. C., Ferrier, R. C., Li, C., Composto, R. J. \& Fakhraai, Z. Out-of-plane orientation alignment and reorientation dynamics of gold nanorods in polymer nanocomposite films. Soft Matter 13, 2207-2215 (2017).

2. Lang, X., Qian, L., Guan, P., Zi, J. \& Chen, M. Localized surface plasmon resonance of nanoporous gold. Appl. Phys. Lett. 98, 1-4 (2011).

3. Detsi, E., Salverda, M., Onck, P. R. \& De Hosson, J. T. M. On the localized surface plasmon resonance modes in nanoporous gold films. J. Appl. Phys. 115, (2014).

4. Bok, H. M., Shuford, K. L., Kim, S., Kim, S. K. \& Park, S. Multiple surface plasmon modes for a colloidal solution of nanoporous gold nanorods and their comparison to smooth gold nanorods. Nano Lett. 8, 2265-2270 (2008).

5. Zhang, Y. \& Fakhraai, Z. Decoupling of surface diffusion and relaxation dynamics of molecular glasses. Proc. Natl. Acad. Sci. 114, 4915-4919 (2017). 